Supporting information for

\title{
Austins-type Meroterpenoids from a Mangrove-derived Penicillium sp.
}

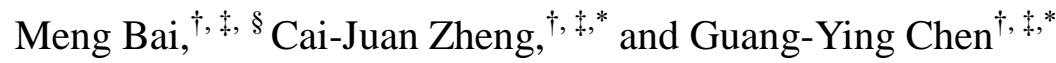

${ }^{\dagger}$ Key Laboratory of Tropical Medicinal Resource Chemistry of Ministry of Education, Hainan Normal University, Haikou, Hainan, 571158, People's Republic of China

${ }^{\ddagger}$ Key Laboratory of Tropical Medicinal Plant Chemistry of Hainan Province, College of Chemistry and Chemical Engineering, Hainan Normal University, Haikou, Hainan, 571158, People's Republic of China

$\S$ Institute of Marine Drugs, Guangxi University of Chinese Medicine, Nanning, Guangxi, 530200, P.R. China. 


\section{List of Supporting Information}

Figure S1. ${ }^{1} \mathrm{H}$ NMR $\left(\mathrm{CD}_{3} \mathrm{OD}, 400 \mathrm{MHz}\right)$ spectrum of 1.

Figure S2. ${ }^{13} \mathrm{C}$ NMR $\left(\mathrm{CD}_{3} \mathrm{OD}, 100 \mathrm{MHz}\right)$ spectrum of $\mathbf{1}$.

Figure S3. DEPT $\left(\mathrm{CD}_{3} \mathrm{OD}, 100 \mathrm{MHz}\right)$ spectrum of $\mathbf{1}$.

Figure S4. HMQC spectrum of $\mathbf{1}$.

Figure S5. HMBC spectrum of $\mathbf{1}$.

Figure S6. COSY spectrum of $\mathbf{1 .}$

Figure S7. NOESY spectrum of $\mathbf{1}$.

Figure S8. HR-ESI-MS spectrum of $\mathbf{1}$.

Figure S9. ${ }^{1} \mathrm{H}$ NMR $\left(\mathrm{CD}_{3} \mathrm{OD}, 400 \mathrm{MHz}\right)$ spectrum of 2.

Figure S10. ${ }^{13} \mathrm{C}$ NMR $\left(\mathrm{CD}_{3} \mathrm{OD}, 100 \mathrm{MHz}\right)$ spectrum of 2.

Figure S11. DEPT $\left(\mathrm{CD}_{3} \mathrm{OD}, 100 \mathrm{MHz}\right)$ spectrum of 2.

Figure S12. HMQC spectrum of 2.

Figure S13. HMBC spectrum of 2.

Figure S14. COSY spectrum of 2.

Figure S15. NOESY spectrum of 2.

Figure S16. HR-ESI-MS spectrum of 2.

Figure S17. ${ }^{1} \mathrm{H}$ NMR $\left(\mathrm{CD}_{3} \mathrm{OD}, 400 \mathrm{MHz}\right)$ spectrum of 3.

Figure S18. ${ }^{13} \mathrm{C}$ NMR $\left(\mathrm{CD}_{3} \mathrm{OD}, 100 \mathrm{MHz}\right)$ spectrum of 3 .

Figure S19. DEPT $\left(\mathrm{CD}_{3} \mathrm{OD}, 100 \mathrm{MHz}\right)$ spectrum of $\mathbf{3}$.

Figure S20. HMQC spectrum of $\mathbf{3}$.

Figure S21. HMBC spectrum of $\mathbf{3}$.

Figure S22. COSY spectrum of $\mathbf{3}$.

Figure S23. NOESY spectrum of 3.

Figure S24. HR-ESI-MS spectrum of 3.

Figure S25. Possible structures of 2 (2a and $\mathbf{2 b}$ ).

Computational details of compound $\mathbf{1}$

Crystal data and structure refinement for $\mathbf{2}$ 


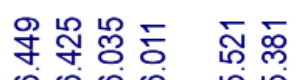

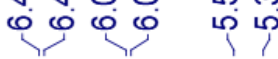

กิ๊

品 兽命
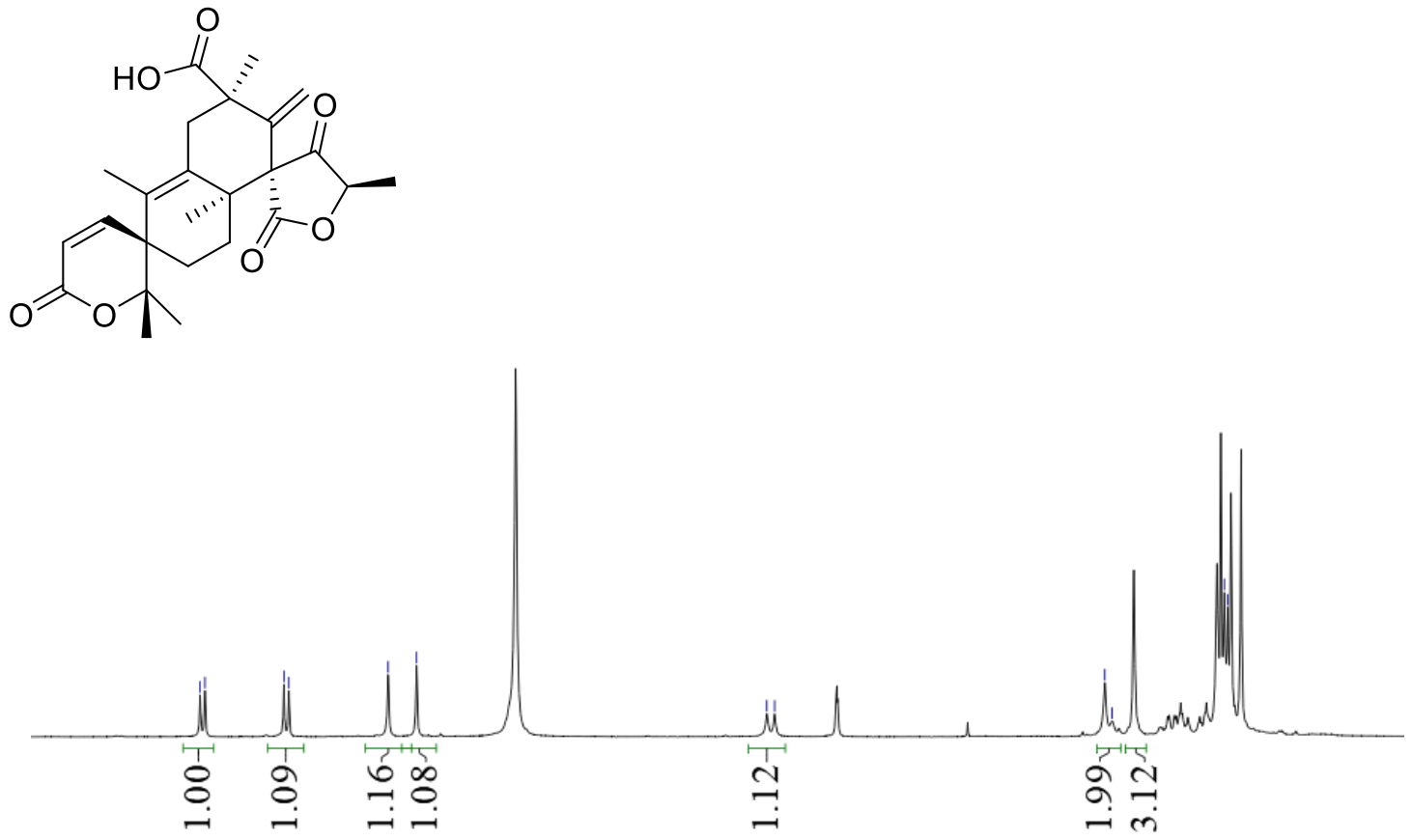

Figure $\mathrm{S} 1 .{ }^{1} \mathrm{H}$ NMR $\left(\mathrm{CD}_{3} \mathrm{OD}, 400 \mathrm{MHz}\right)$ spectrum of $\mathbf{1}$.

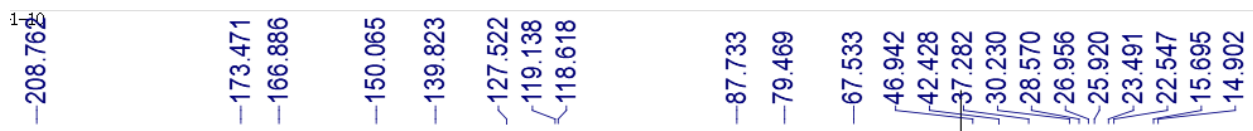

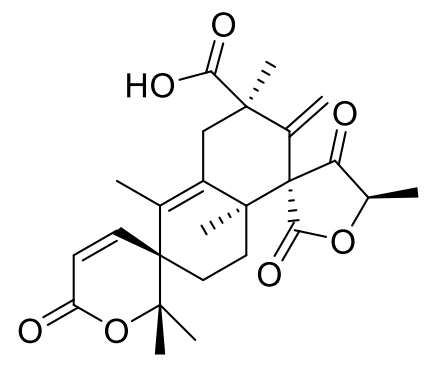




\begin{tabular}{|c|c|c|c|}
\hline $\begin{array}{l}0 \\
0 \\
0 \\
10 \\
10\end{array}$ & 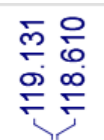 & 足 & 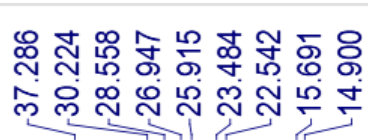 \\
\hline
\end{tabular}

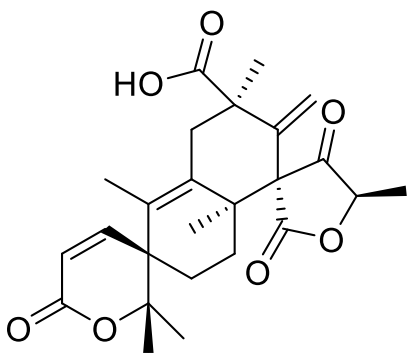

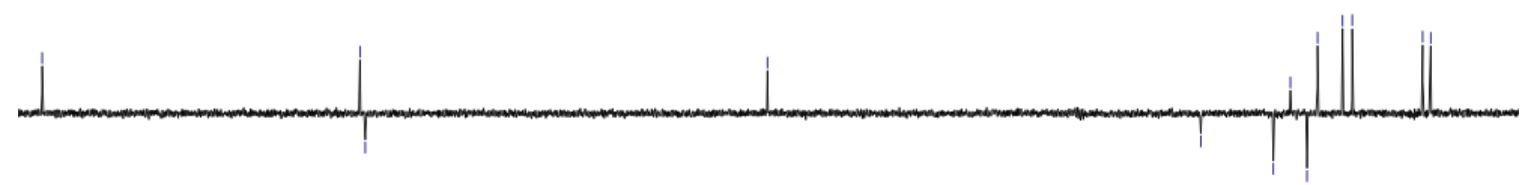

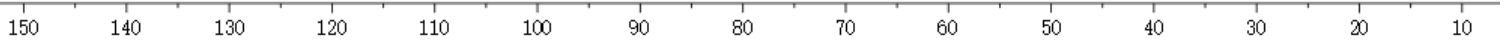

Figure S3. DEPT $\left(\mathrm{CD}_{3} \mathrm{OD}, 100 \mathrm{MHz}\right)$ spectrum of $\mathbf{1}$.

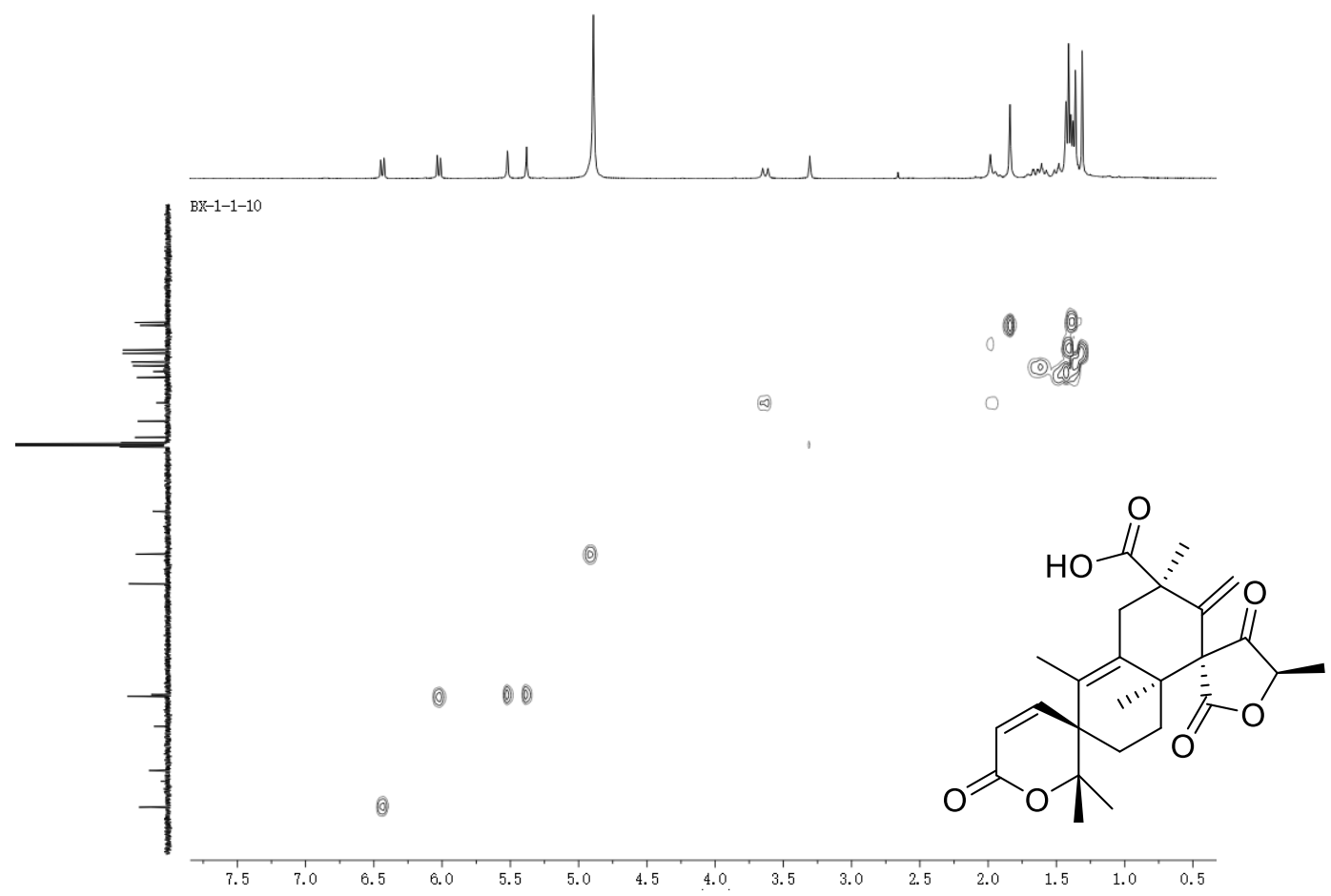

Figure S4. HMQC spectrum of $\mathbf{1}$. 


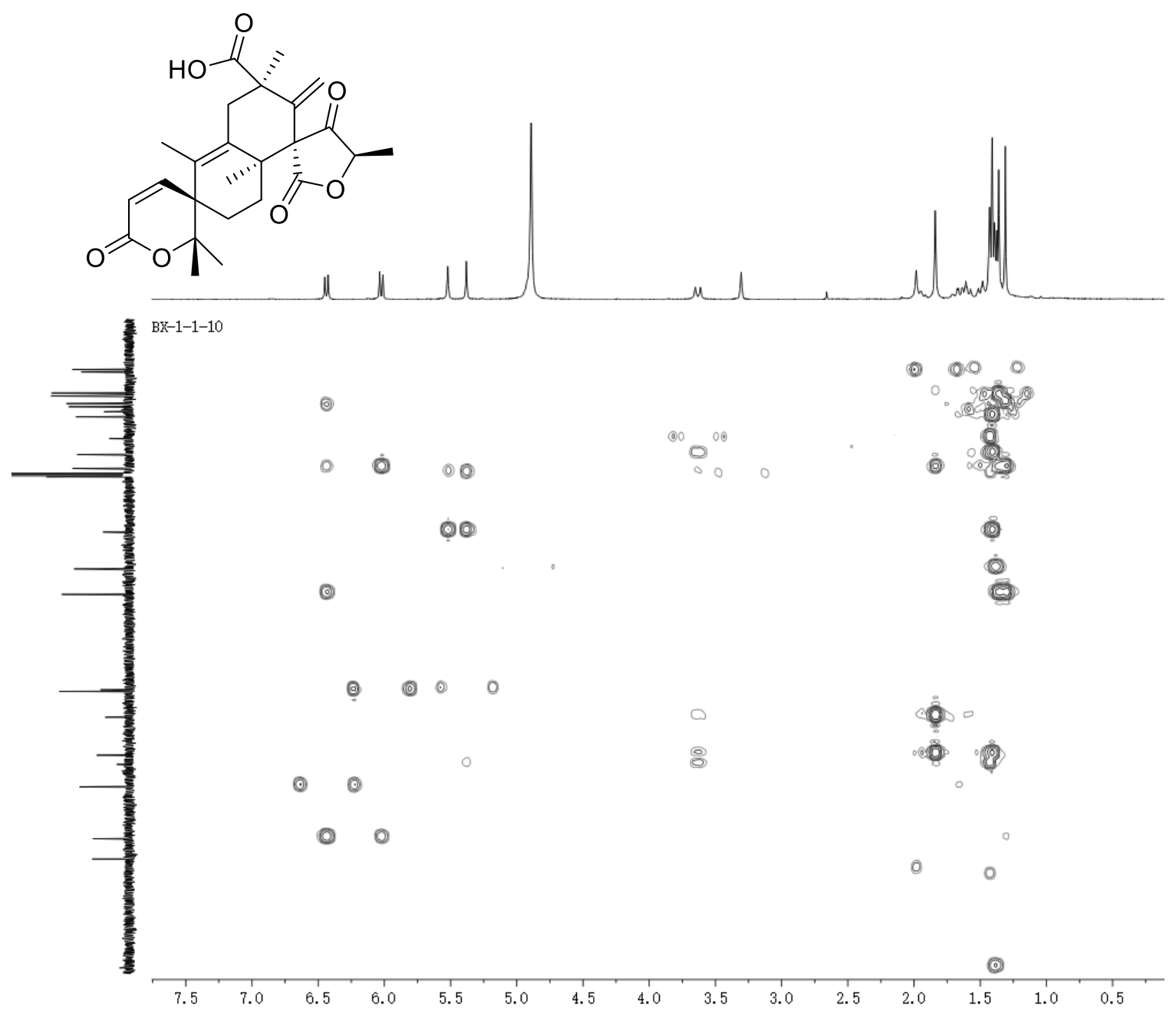

Figure S5. HMBC spectrum of $\mathbf{1}$.

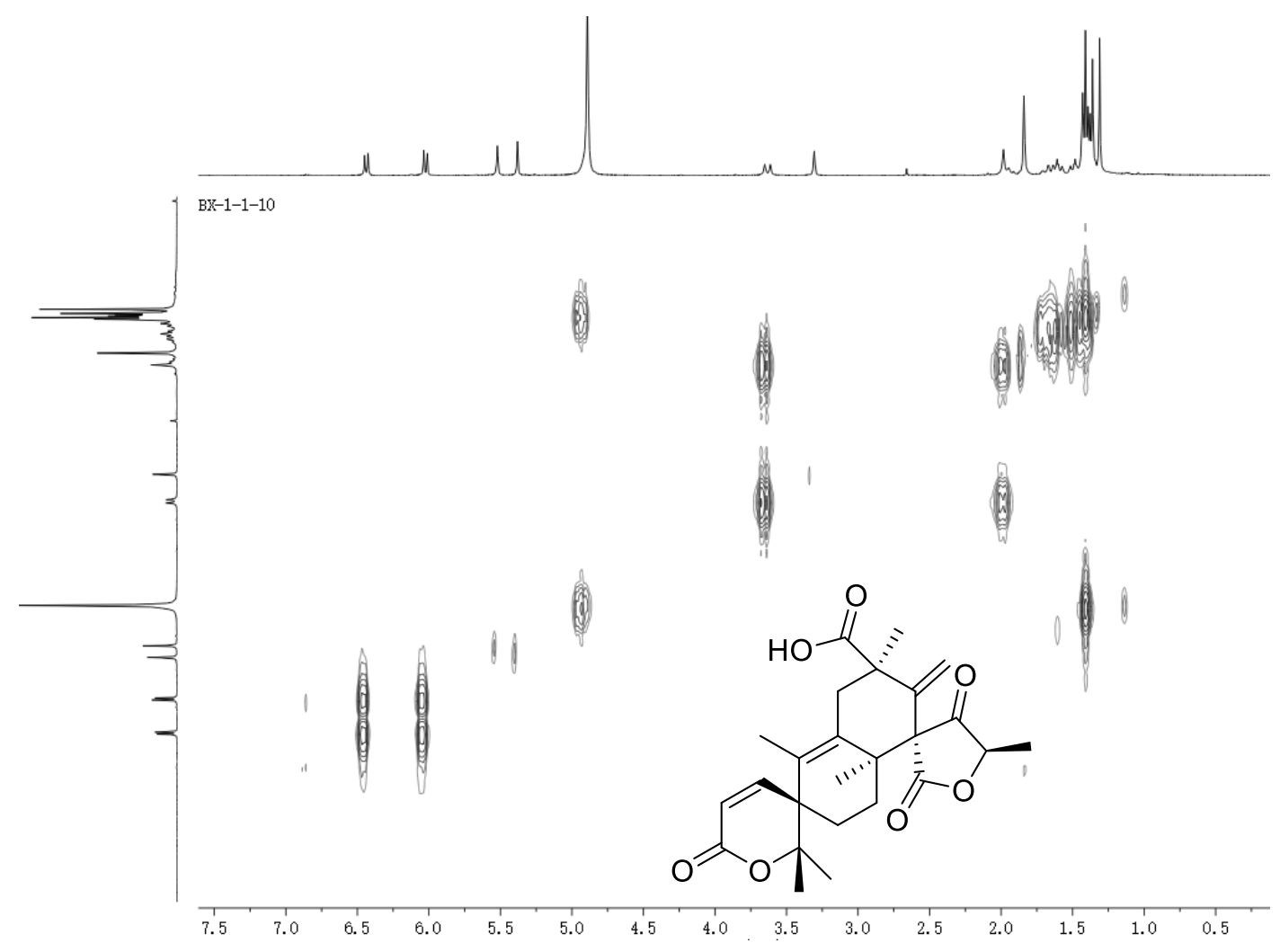

Figure S6. COSY spectrum of 1. 


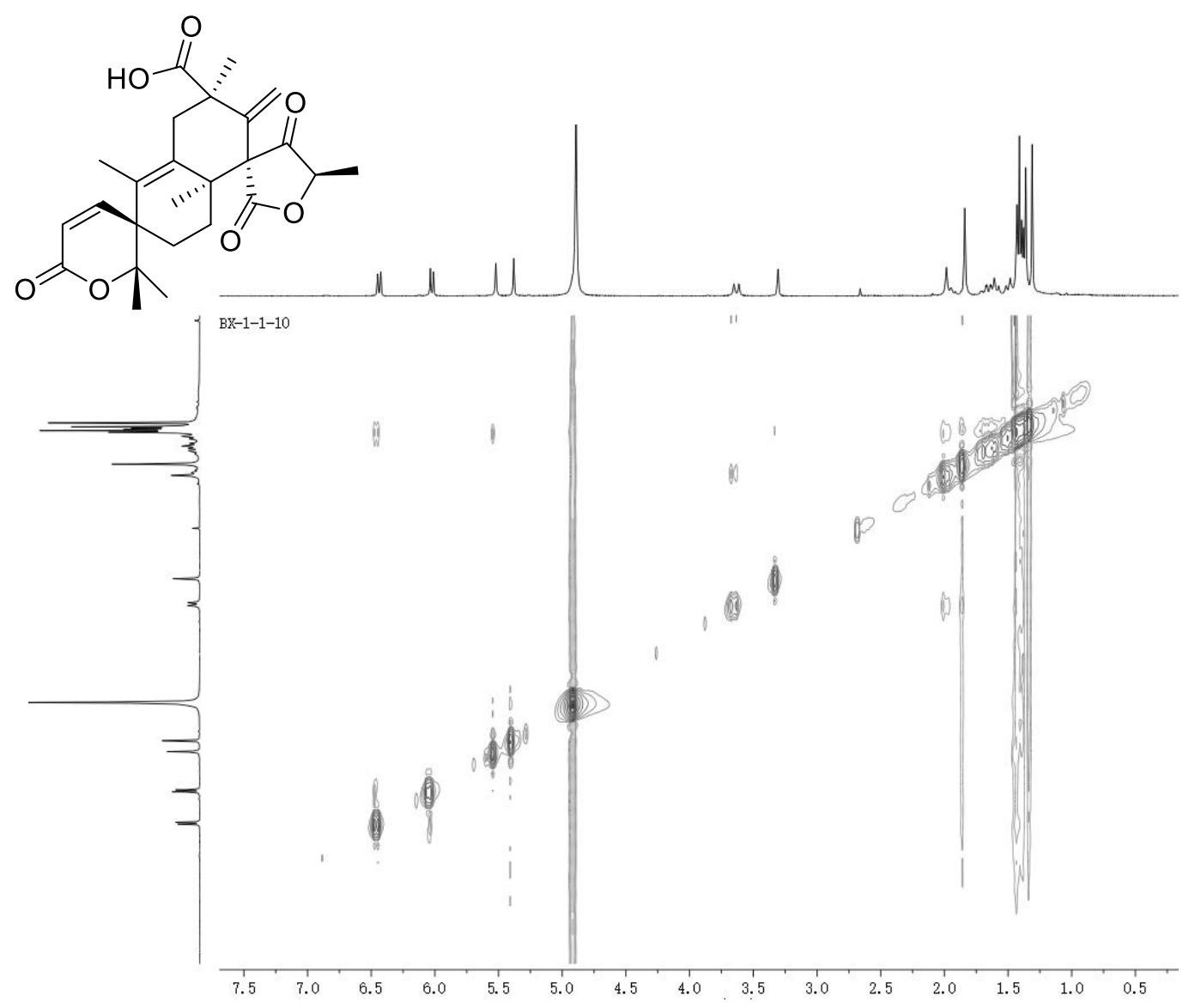

Figure S7. NOESY spectrum of $\mathbf{1}$.

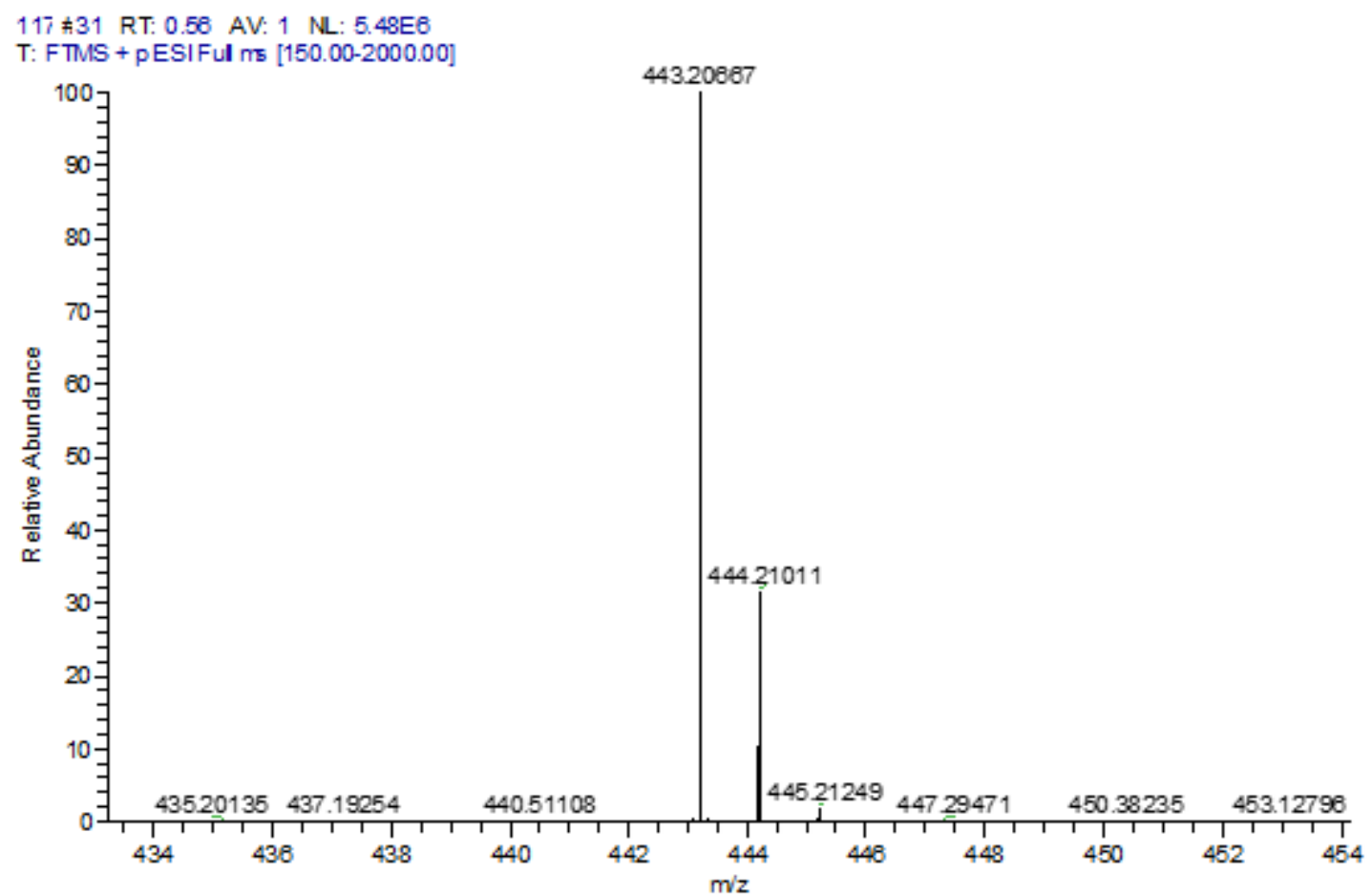

Figure S8. HRESIMS spectrum of $\mathbf{1}$. 


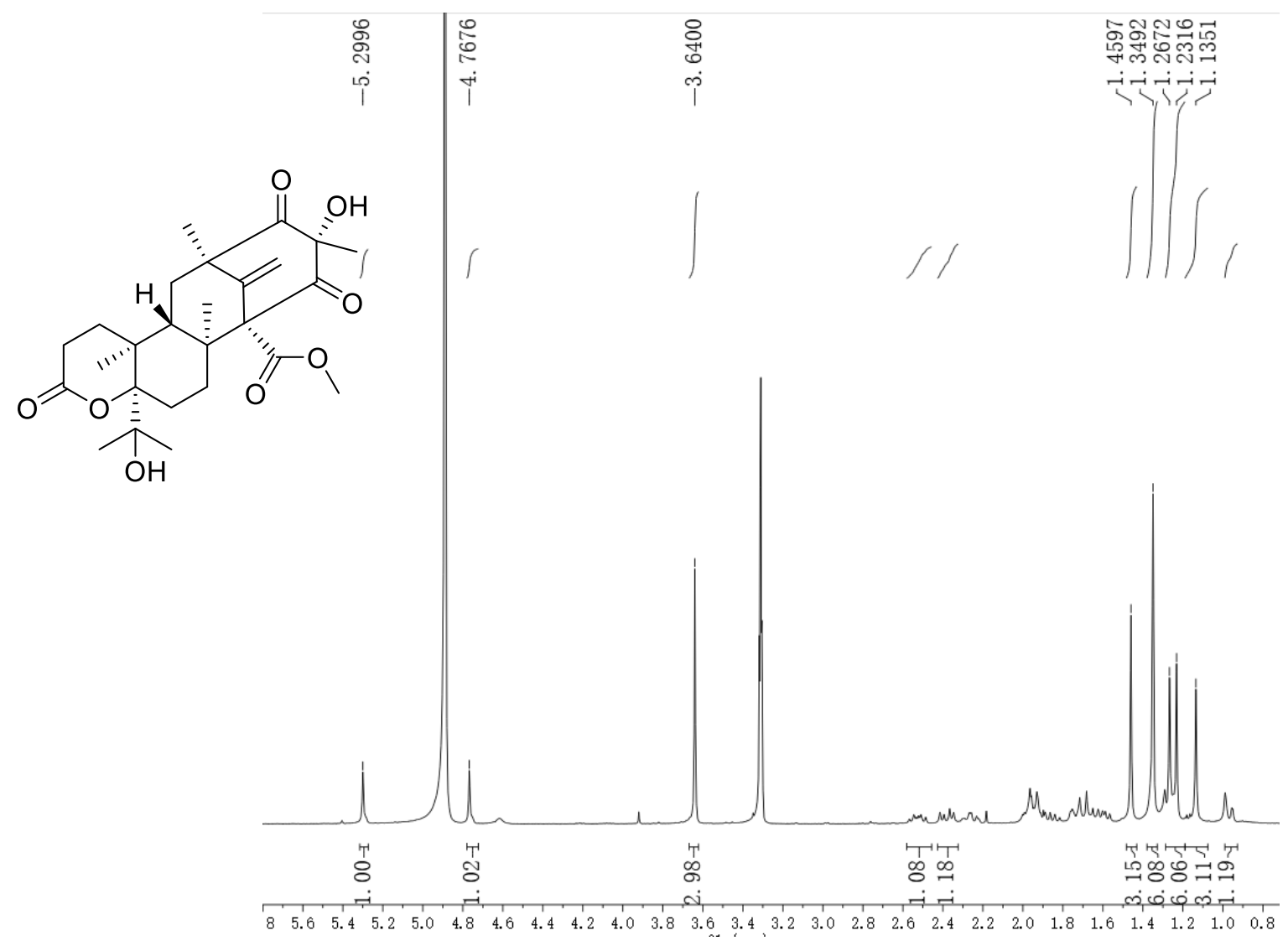

Figure S9. ${ }^{1} \mathrm{H}$ NMR $\left(\mathrm{CD}_{3} \mathrm{OD}, 400 \mathrm{MHz}\right)$ spectrum of 2.

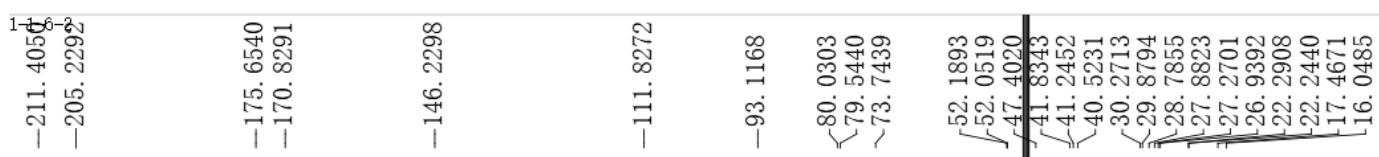

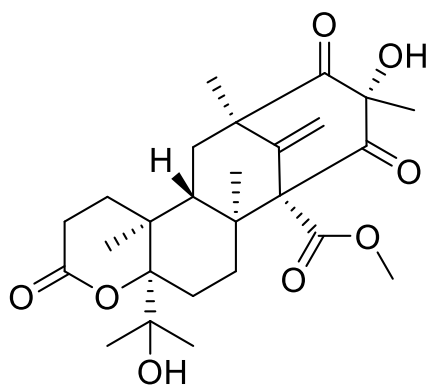

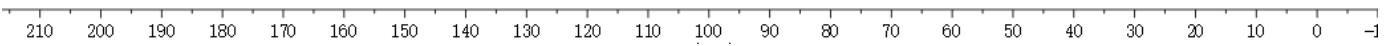


Figure S10. ${ }^{13} \mathrm{C}$ NMR $\left(\mathrm{CD}_{3} \mathrm{OD}, 100 \mathrm{MHz}\right)$ spectrum of 2.

ồ

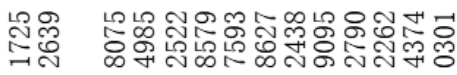

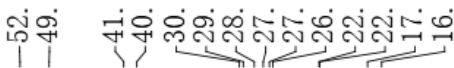<smiles>C=C1[C@]2(C(=O)OC)C(=O)[C@@](C)(O)C(=O)[C@]1(C)C[C@@H]1[C@@]2(C)CC[C@]2(C(C)(C)O)OC(=O)CC[C@]12C</smiles>

$\mathrm{OH}$

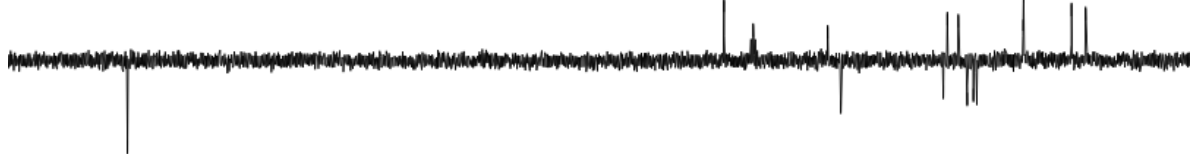

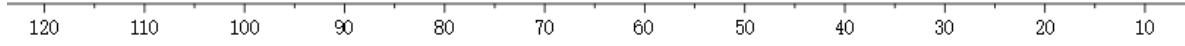

Figure S11. DEPT $\left(\mathrm{CD}_{3} \mathrm{OD}, 100 \mathrm{MHz}\right)$ spectrum of 2.

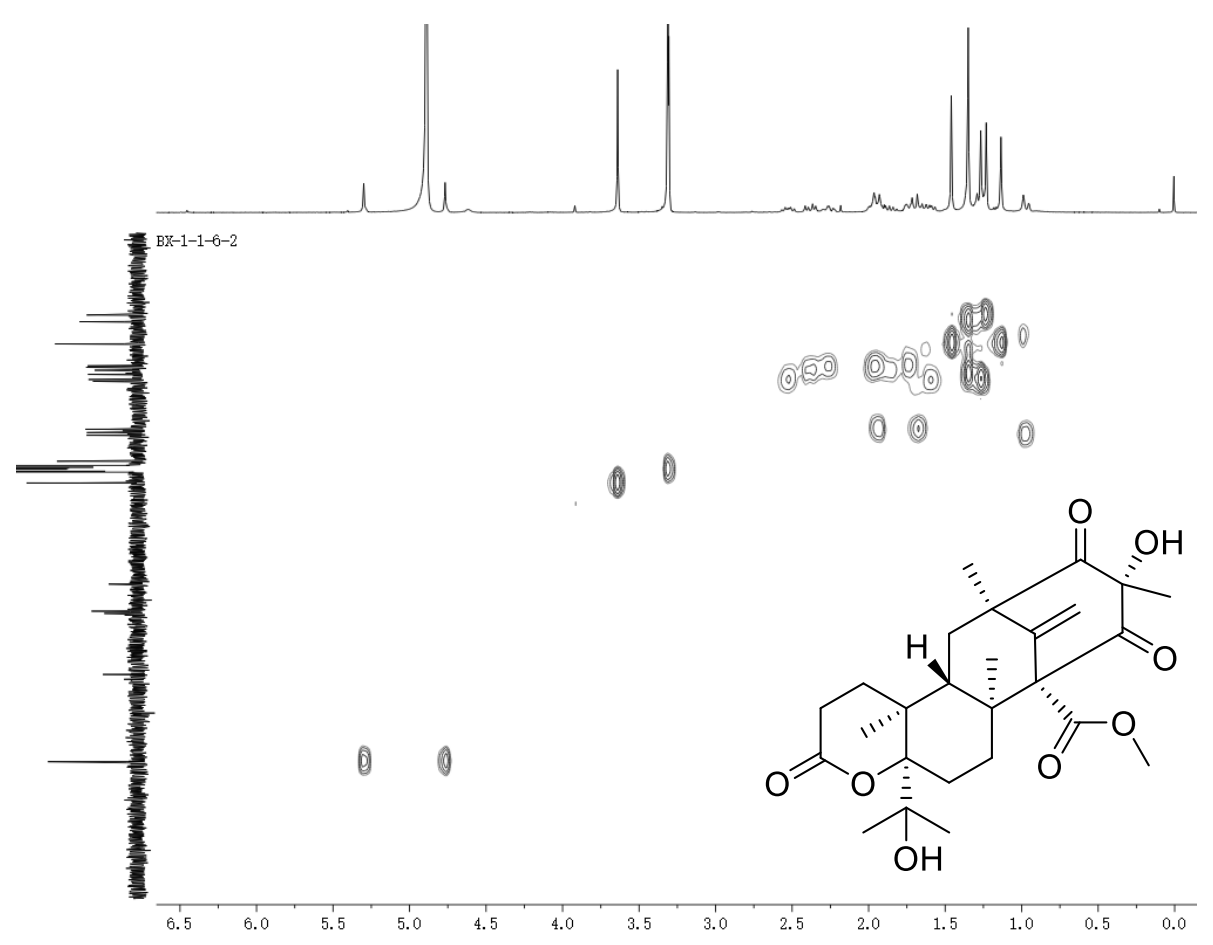

Figure S12. HMQC spectrum of 2. 


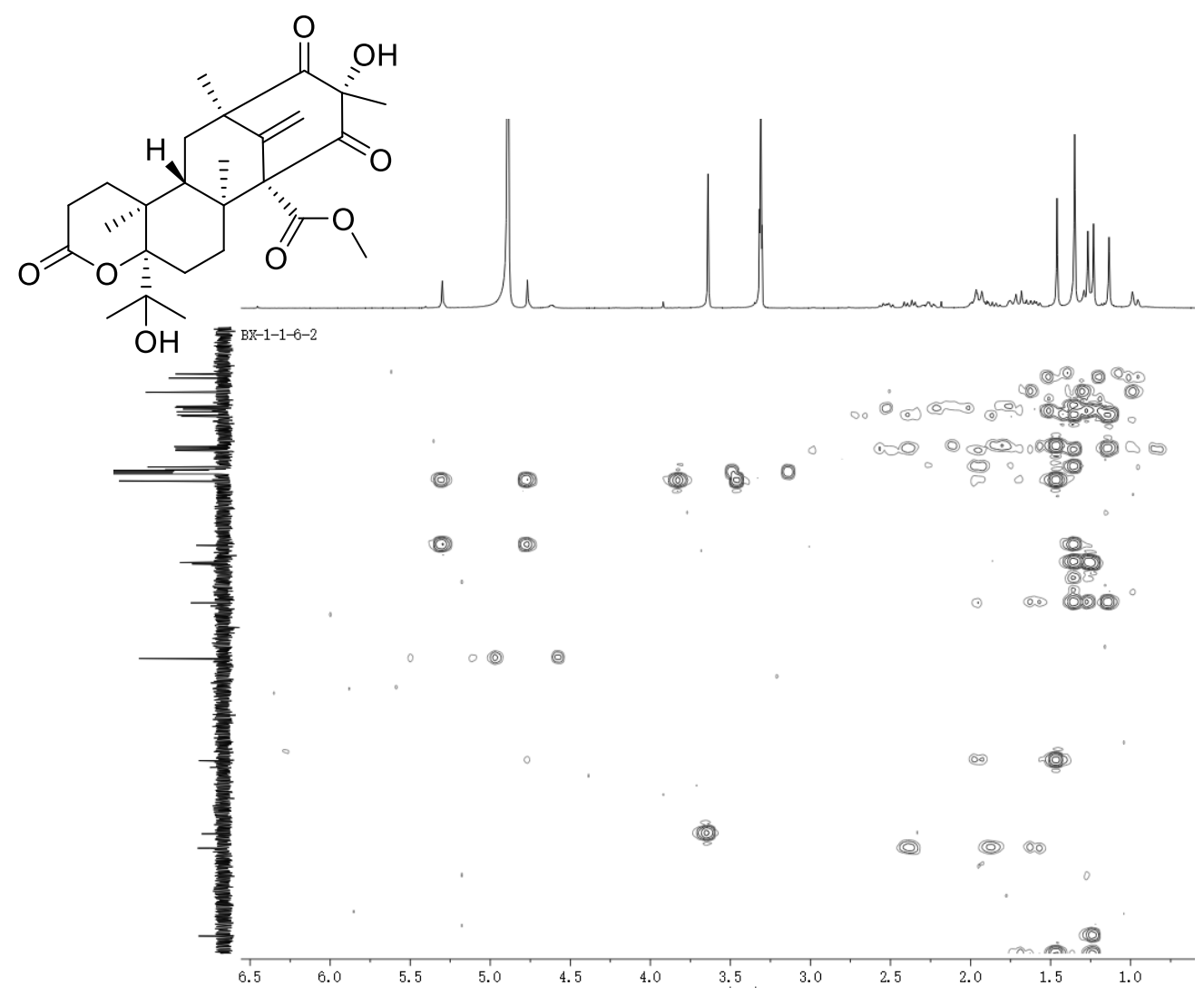

Figure S13. HMBC spectrum of 2.

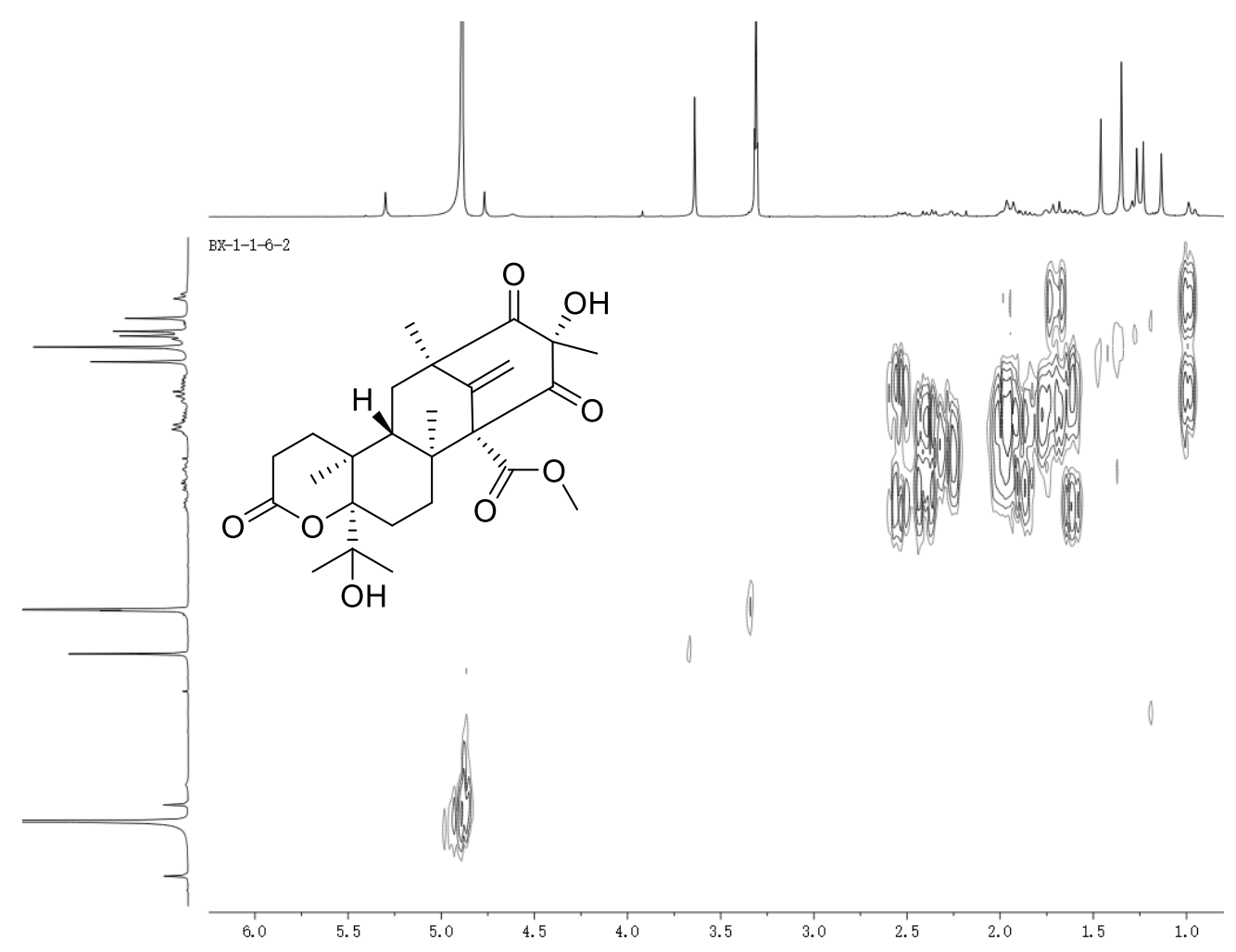

Figure S14. COSY spectrum of 2. 


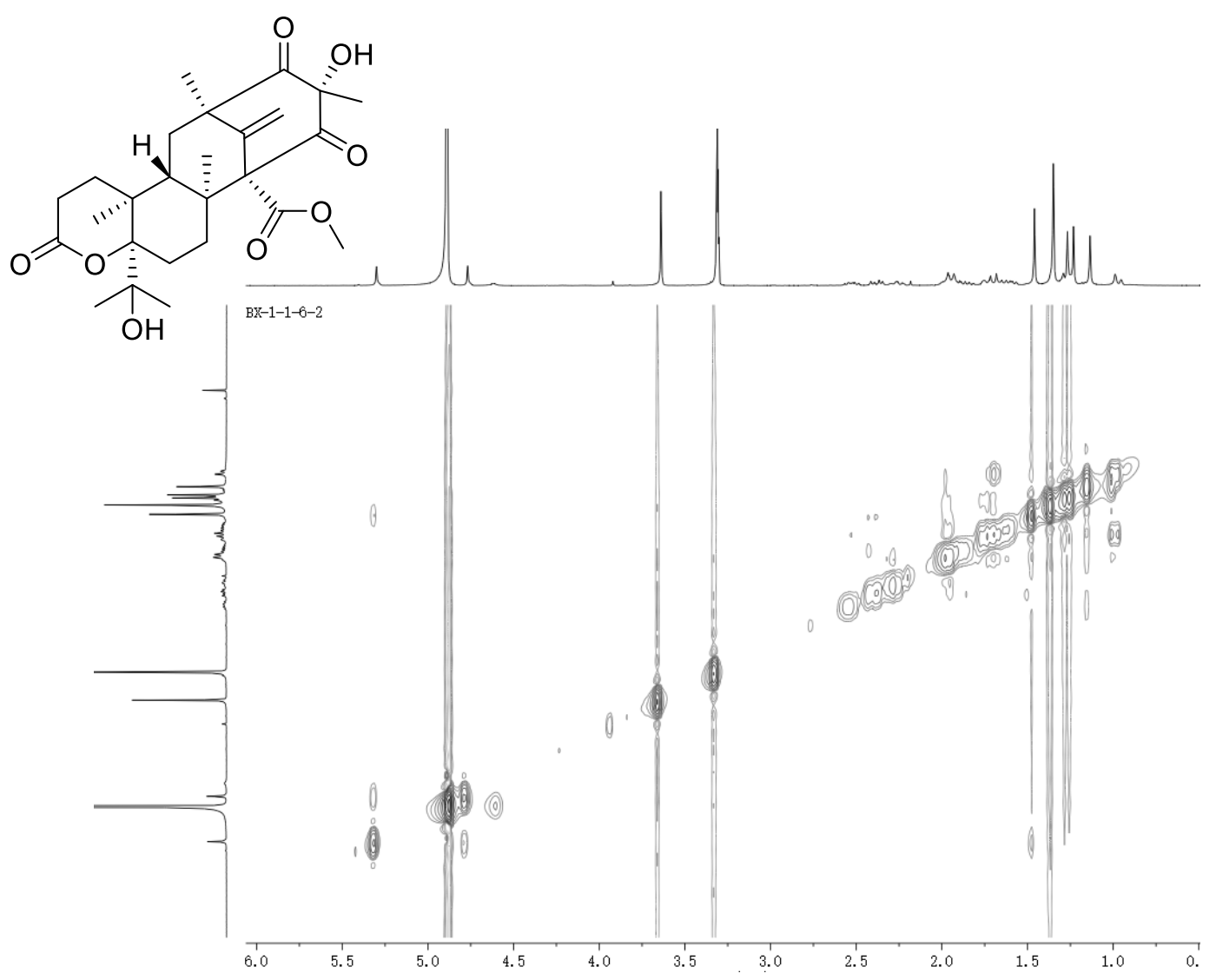

Figure S15. NOESY spectrum of 2.

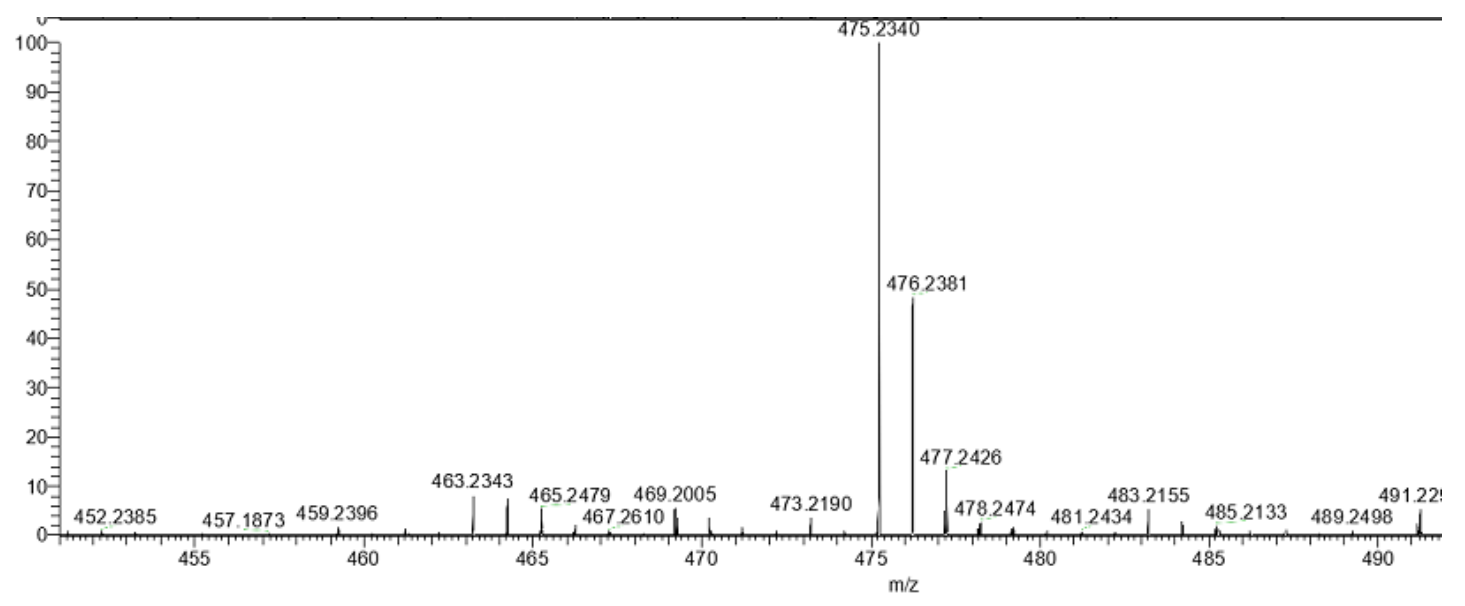

Figure S16. HR-ESI-MS spectrum of 2. 


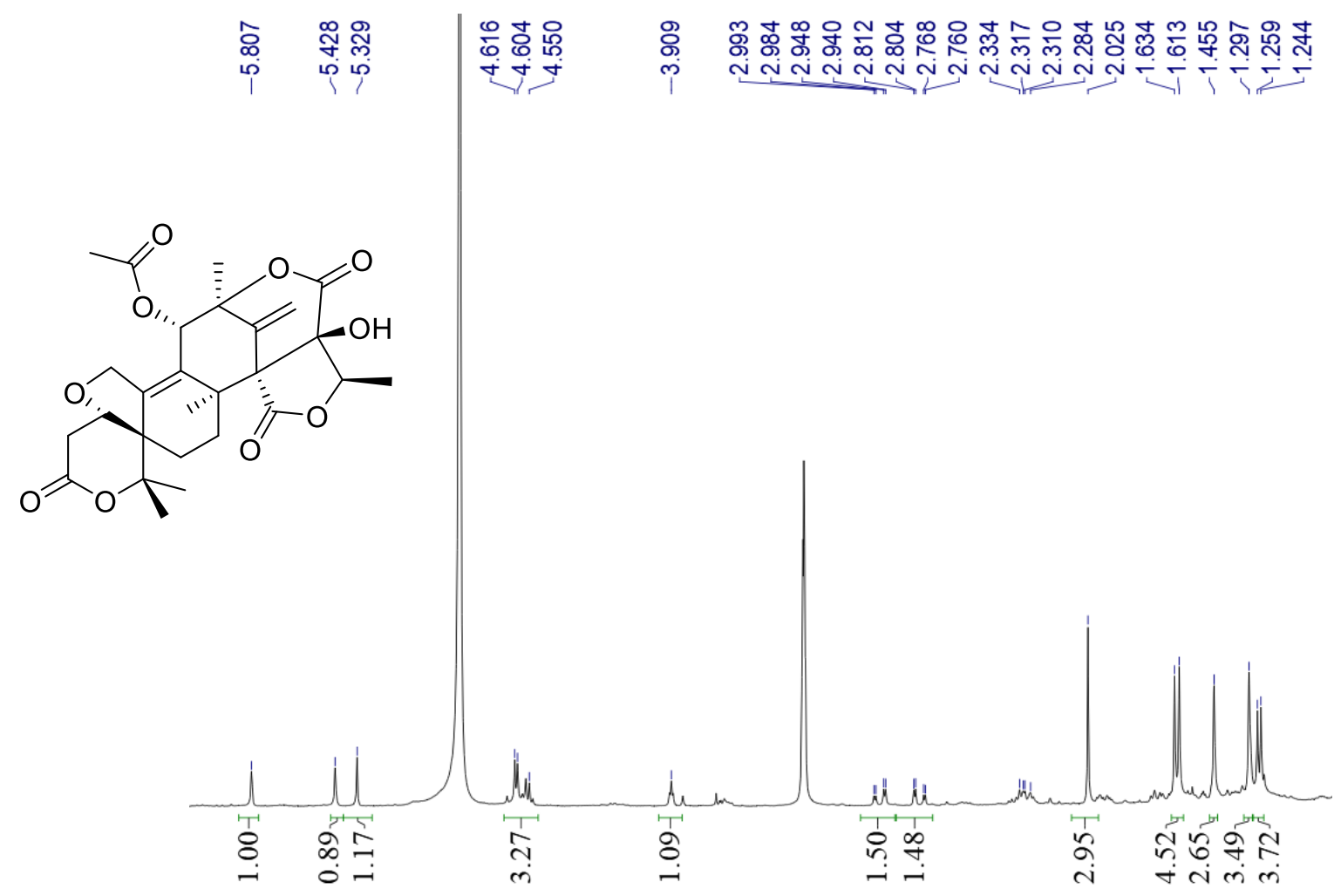

Figure S17. ${ }^{1} \mathrm{H}$ NMR $\left(\mathrm{CD}_{3} \mathrm{OD}, 400 \mathrm{MHz}\right)$ spectrum of 3.
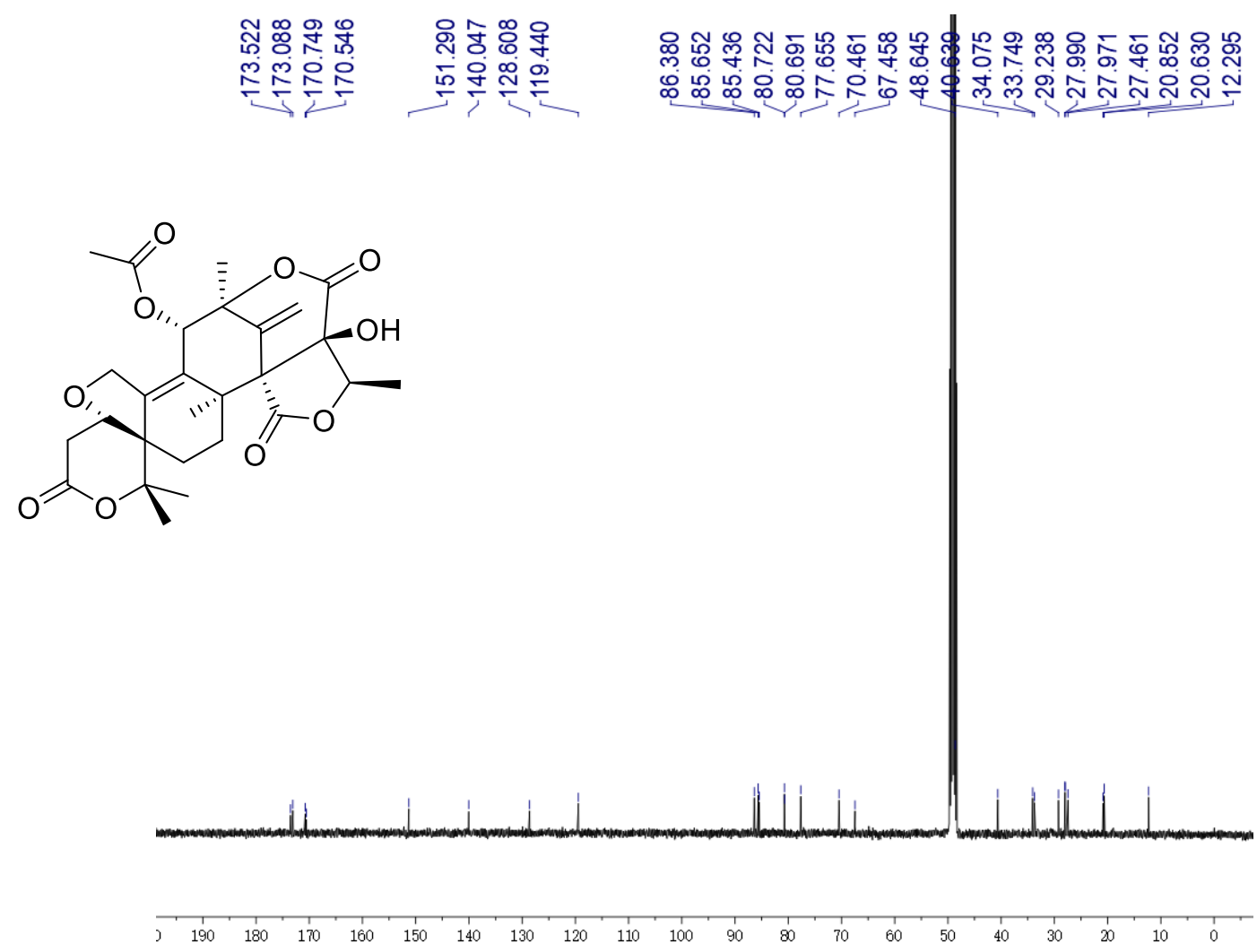

Figure S18. ${ }^{13} \mathrm{C}$ NMR $\left(\mathrm{CD}_{3} \mathrm{OD}, 100 \mathrm{MHz}\right)$ spectrum of 3. 


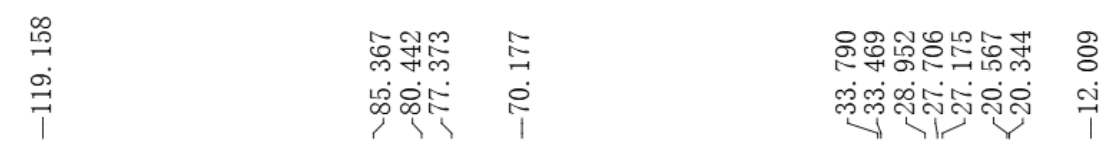

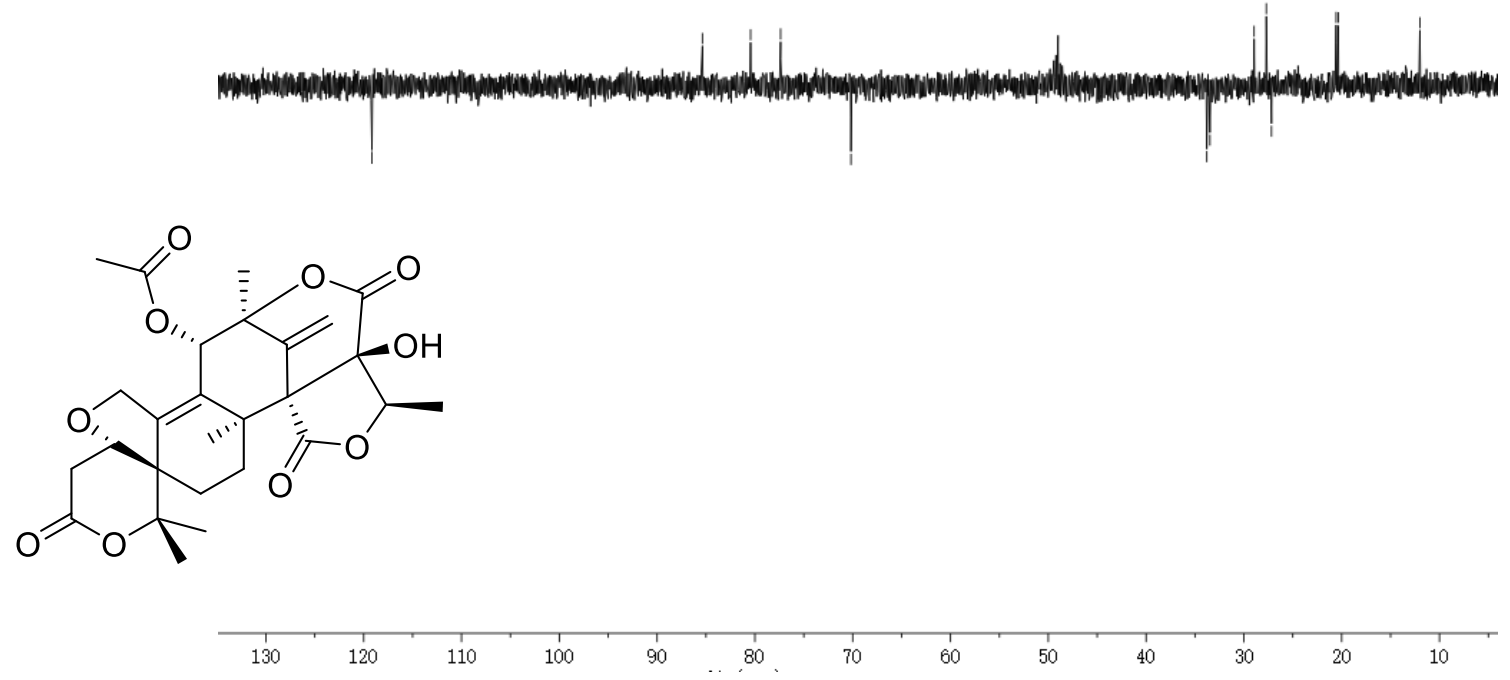

Figure S19. DEPT $\left(\mathrm{CD}_{3} \mathrm{OD}, 100 \mathrm{MHz}\right)$ spectrum of $\mathbf{3}$.

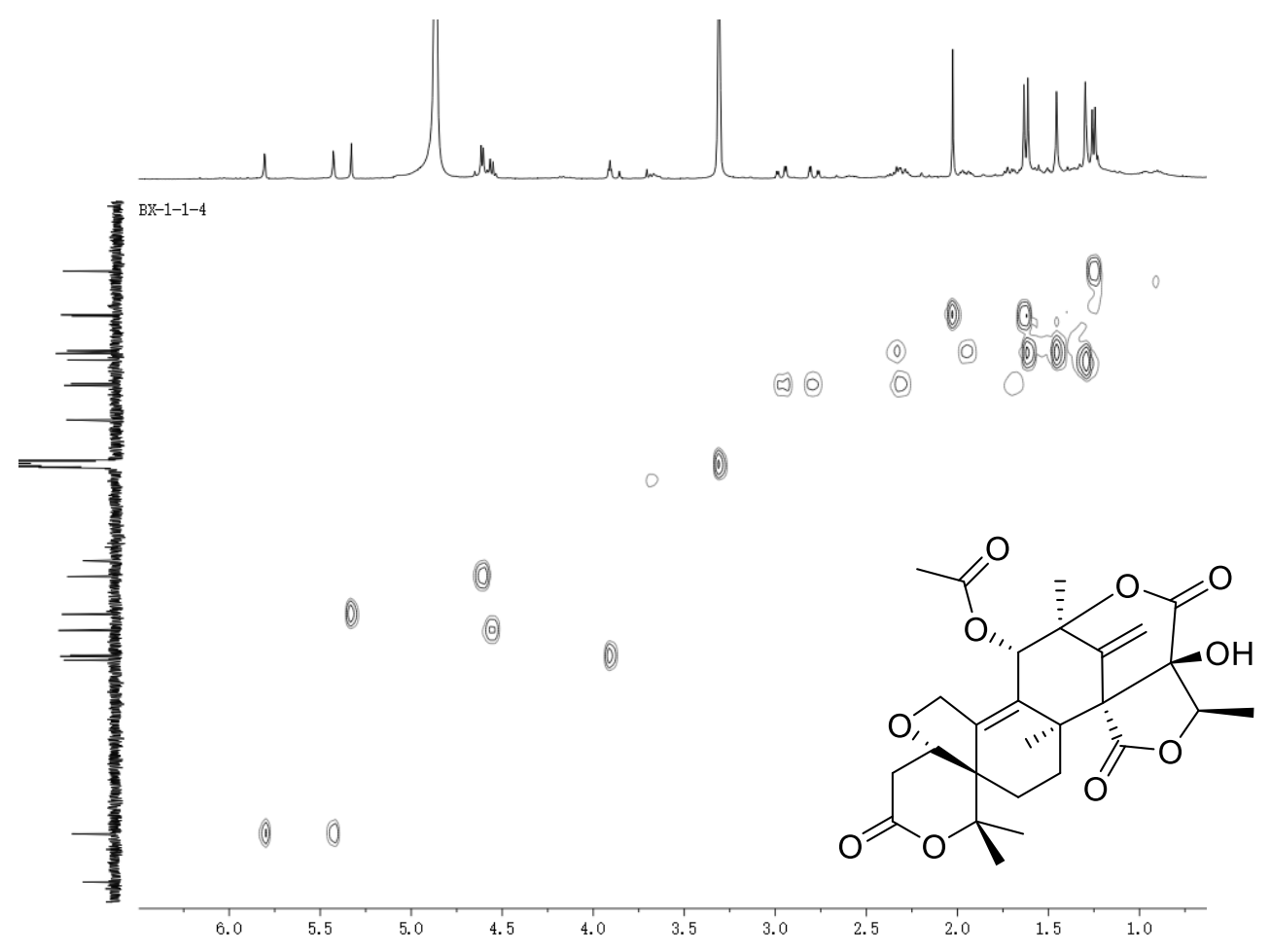

Figure S20. HMQC spectrum of $\mathbf{3}$. 


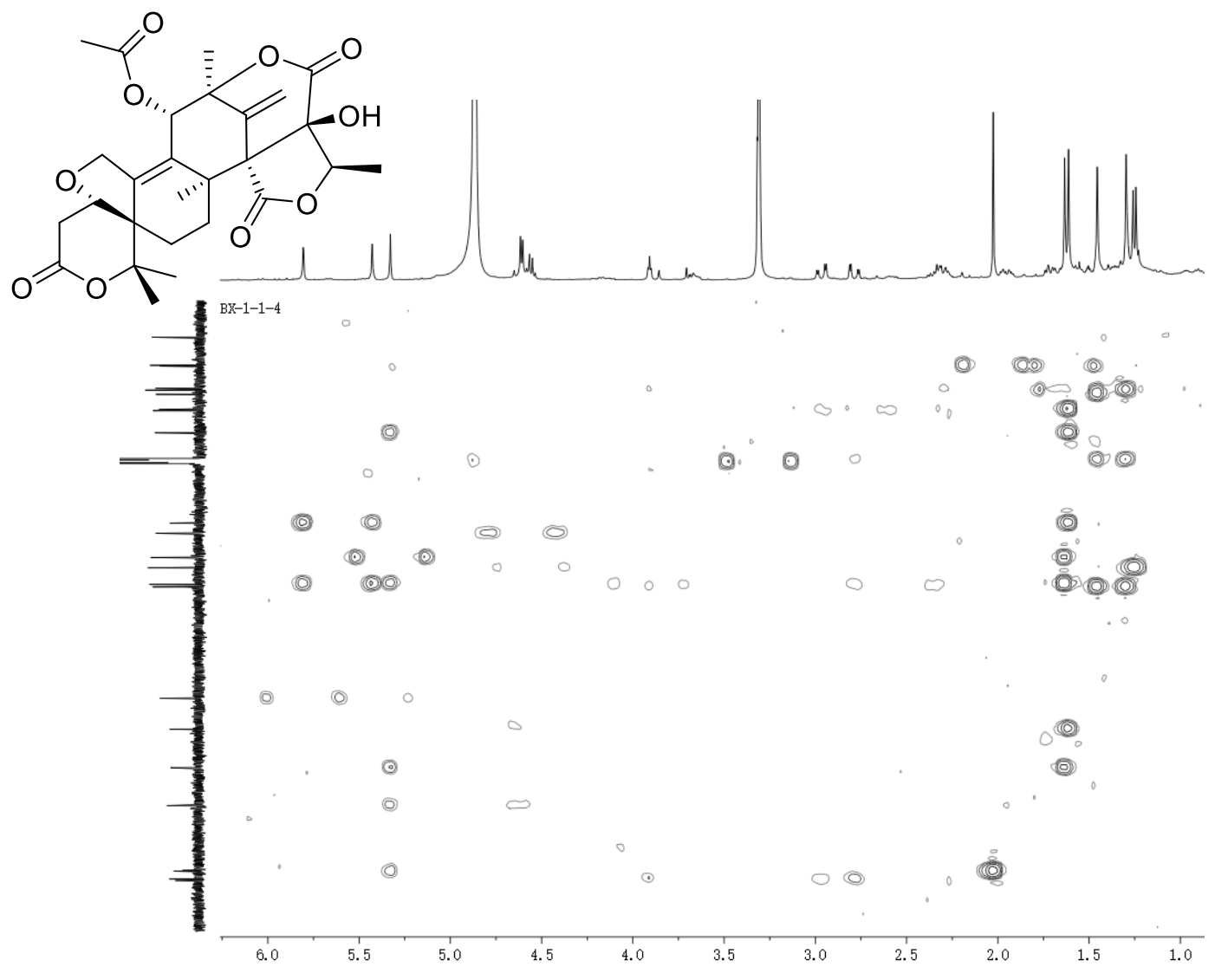

Figure S21. HMBC spectrum of 3 .

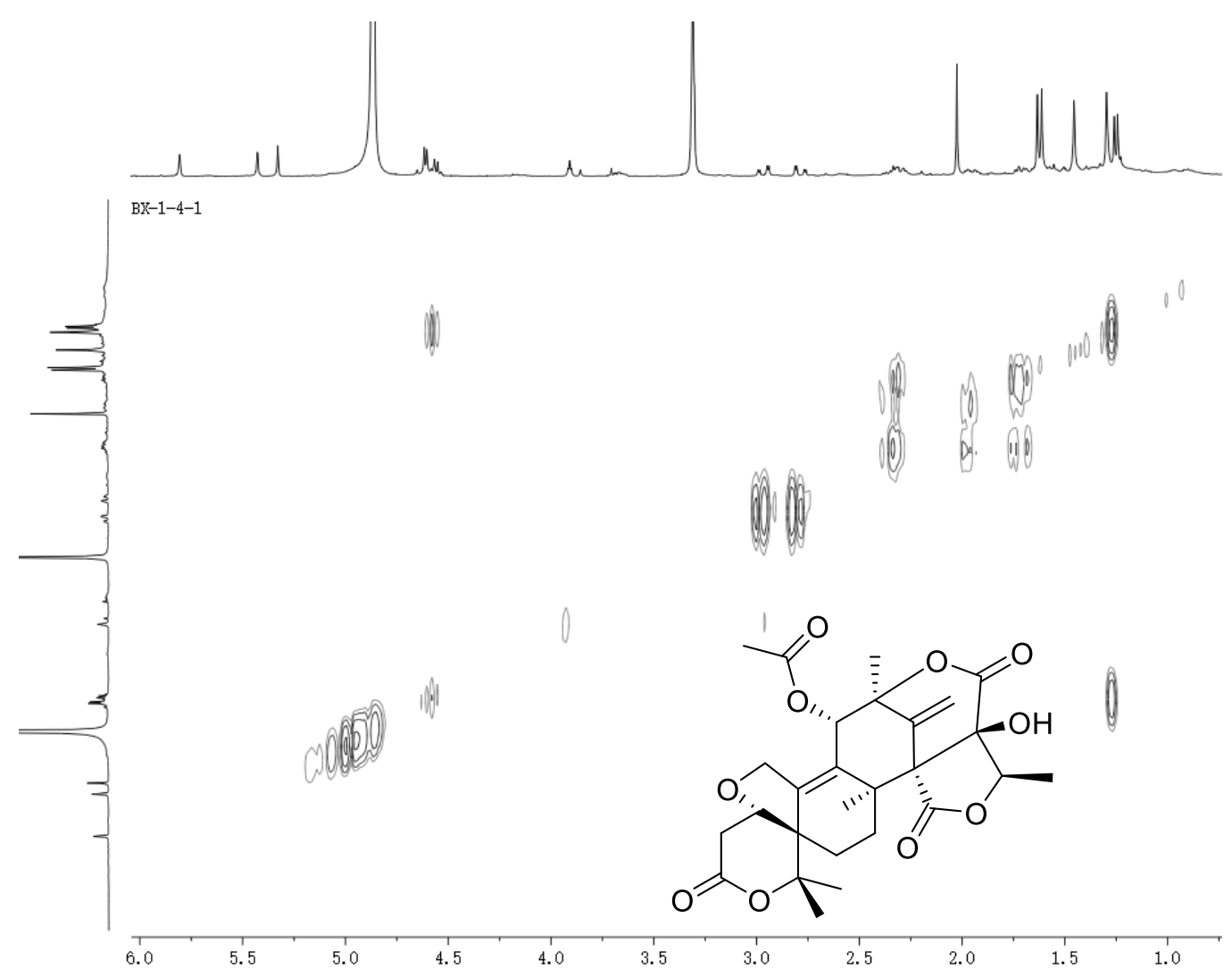

Figure S22. COSY spectrum of $\mathbf{3}$. 


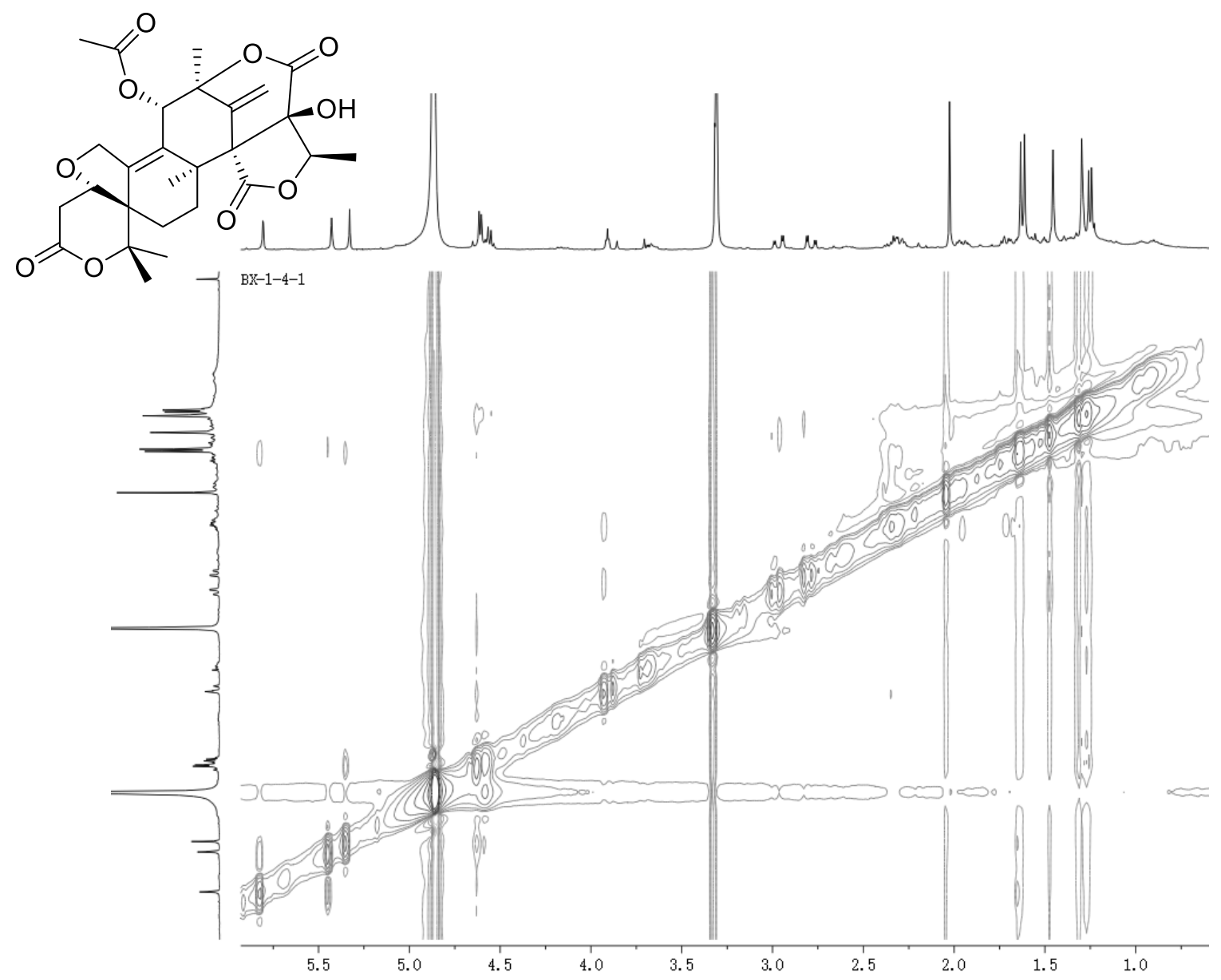

Figure S23. NOESY spectrum of 3.

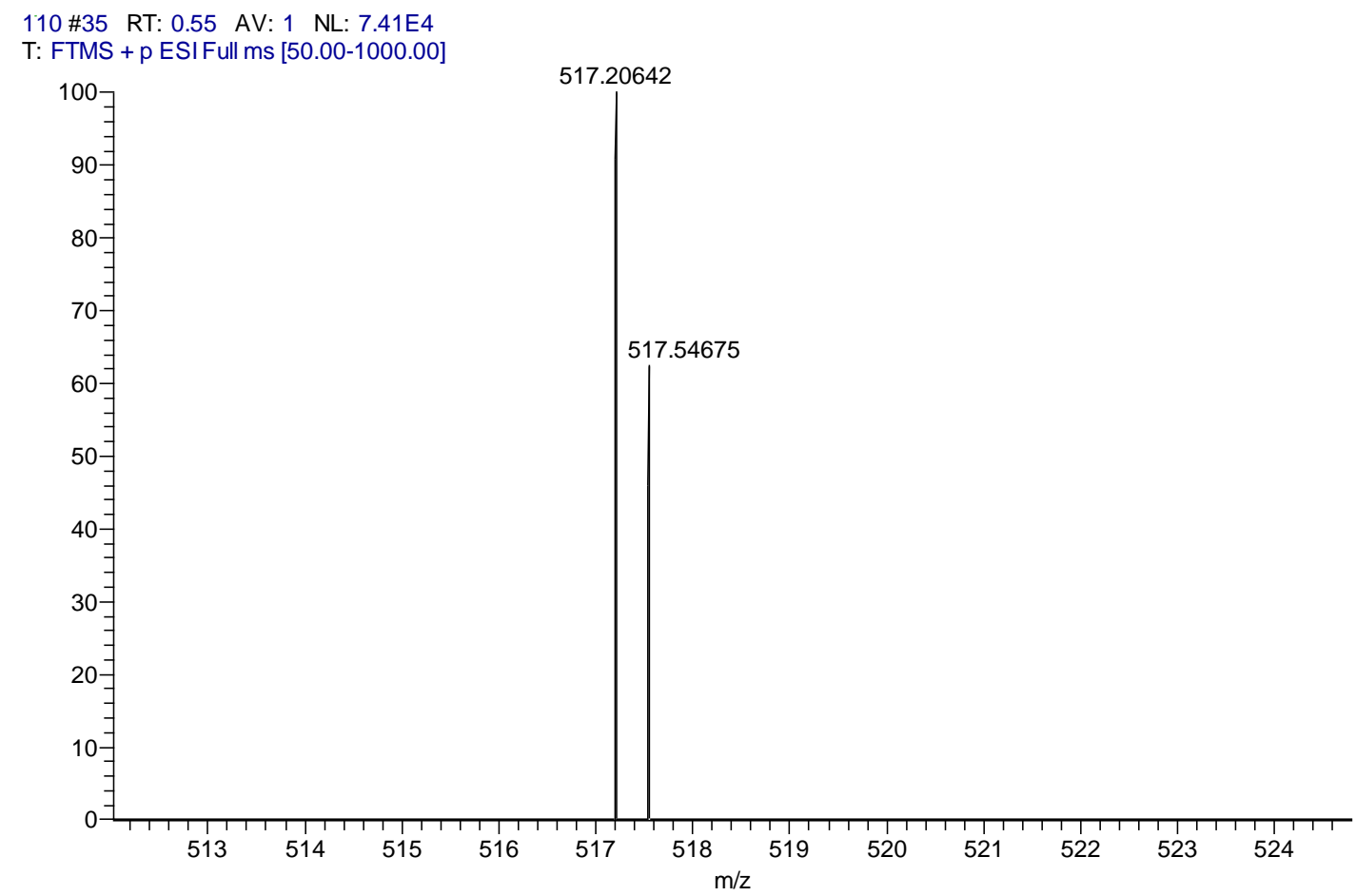

Figure S24. HR-ESI-MS spectrum of 3. 


\section{Computational details of compound 1}

The initial geometries of penicianstinoid C (1) was generated based on a conformational search by MMFF force filed. Then, $\mathbf{1 3}$ low-energy conformers within a $10 \mathrm{kcal} / \mathrm{mol}$ energy difference were selected and further optimized at B3LYP/6-311++G(2d,p) method in vacuum by Gaussian 09. The stability of optimized structure was confirmed by a successive frequency calculation at the same method. Finally, the ECD calculation was performed on these conformers by TDDFT calculation at B3LYP/6-311++G(2d,p) method. The final ECD spectra of penicianstinoid C (1) was summed based on a Boltzmann population analysis at 298K.

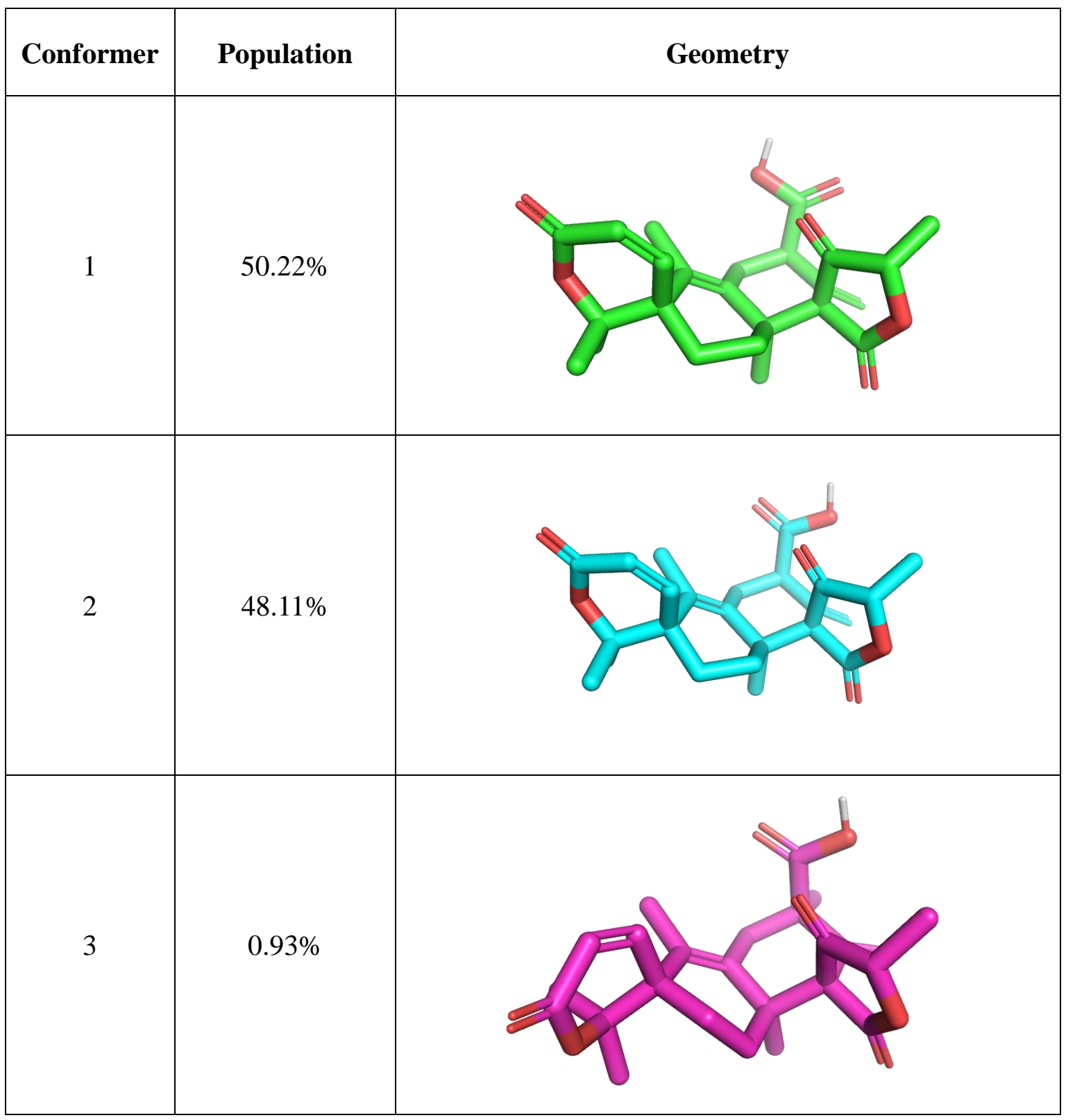

*Other 10 conformers were not shown because their populations were less than $0.5 \%$. 


\section{Coordinates of optimized conformers}

(1). Conformer1, Pop $=50.22 \%$ B3LYP/6-311++g(2d,p), $E=-1497.71726087$ au 62

$\mathrm{C}$

$\mathrm{C}$

$\mathrm{O}$

C

C

C

C

C

C

C

$\mathrm{C}$

C

$\mathrm{C}$

$\mathrm{C}$

$\mathrm{C}$

$\mathrm{C}$

$\mathrm{C}$

$\mathrm{O}$

C

$\mathrm{O}$

C

C

O

$\mathrm{O}$

C

C

C

C

$\mathrm{O}$

$\mathrm{O}$

C

C

$\mathrm{H}$

$\mathrm{H}$

$\mathrm{H}$

$\mathrm{H}$

$\mathrm{H}$

$\mathrm{H}$

$\mathrm{H}$

$\begin{array}{lcc}-3.72420100 & -0.03008300 & 2.03039400 \\ -4.74422200 & 0.65078700 & 1.20659200\end{array}$

$\begin{array}{lll}-4.59688600 & 0.56691600 & -0.14089900\end{array}$

$\begin{array}{lll}-3.77149000 & -0.49150900 & -0.73454200\end{array}$

$-2.37165000 \quad-0.59060600-0.00374400$

$\begin{array}{lll}-2.62744000 & -0.56473000 & 1.48829600\end{array}$

$\begin{array}{lll}-1.73014000 & -1.95076300 & -0.38260200\end{array}$

$-0.24444400-2.00949100-0.06512300$

$\begin{array}{lll}0.54332000 & -0.95430000 & -0.86145400\end{array}$

$-0.15926900 \quad 0.40133800 \quad-0.79865600$

$\begin{array}{lll}-1.42121300 & 0.58941000 & -0.35391900\end{array}$

$\begin{array}{lll}1.98571500 & -0.78732800 & -0.19933100\end{array}$

$2.81051000 \quad 0.31481800 \quad-0.88980000$

$\begin{array}{lll}2.13055500 & 1.68432100 & -0.96961900\end{array}$

$0.65194700 \quad 1.52141900-1.41925400$

$\begin{array}{lll}1.88965000 & -0.47359700 & 1.30776800\end{array}$

$2.79941600 \quad-1.43281400 \quad 2.06211800$

$3.20619900 \quad-2.40702200 \quad 1.07967000$

$\begin{array}{lll}2.76186300 & -2.12082100 & -0.17280300\end{array}$

$\begin{array}{lll}-5.63753000 & 1.31799500 & 1.67075400\end{array}$

$\begin{array}{lll}-4.59801100 & -1.78576500 & -0.62063600\end{array}$

$\begin{array}{lll}-3.66481300 & -0.10078300 & -2.20797100\end{array}$

$2.99028600 \quad-2.86147600 \quad-1.08644200$

$\begin{array}{lll}1.21159000 & 0.36334900 & 1.84437300\end{array}$

$\begin{array}{lll}4.01908700 & 0.07761500 & -1.40087700\end{array}$

$\begin{array}{lll}-1.97046100 & 1.98596000 & -0.12745200\end{array}$

$\begin{array}{lll}2.29399400 & 2.43602700 & 0.36622300\end{array}$

$\begin{array}{lll}2.81833500 & 2.62715600 & -1.99166800\end{array}$

$\begin{array}{lll}3.26339400 & 2.36279500 & 1.07894900\end{array}$

$\begin{array}{lll}1.28561100 & 3.30291800 & 0.60589200\end{array}$

$\begin{array}{lll}0.68793700 & -1.40738700 & -2.33750800\end{array}$

$\begin{array}{lll}4.00453700 & -0.75553400 & 2.70107200\end{array}$

$\begin{array}{lll}-3.89108200 & 0.00187100 & 3.10089300\end{array}$

$\begin{array}{lll}-1.85414400 & -0.97553500 & 2.13180700\end{array}$

$\begin{array}{lll}-1.87617100 & -2.14101300 & -1.44812600\end{array}$

$\begin{array}{lll}-2.24661600 & -2.75717600 & 0.14333500\end{array}$

$\begin{array}{lll}-0.10816100 & -1.85678500 & 1.00996600\end{array}$

$\begin{array}{lll}0.14100400 & -3.00872100 & -0.28882500\end{array}$

$\begin{array}{lll}0.15389400 & 2.48089300 & -1.32946500\end{array}$

$\mathrm{H}$

0.69049100

$1.31787300 \quad-2.49657600$

$\mathrm{H}$

2.21038500

$-1.96209000$

2.81756700 


$\begin{array}{lrrr}\mathrm{H} & -4.18482600 & -2.58894700 & -1.23193700 \\ \mathrm{H} & -5.60739700 & -1.57822400 & -0.98069000 \\ \mathrm{H} & -4.67493100 & -2.14211100 & 0.40759000 \\ \mathrm{H} & -4.66746300 & -0.01244500 & -2.63061500 \\ \mathrm{H} & -3.15463700 & 0.85018700 & -2.34553000 \\ \mathrm{H} & -3.12663900 & -0.86195300 & -2.77603200 \\ \mathrm{H} & 4.59465000 & 0.85598300 & -1.88438100 \\ \mathrm{H} & 4.48367200 & -0.89975200 & -1.37670400 \\ \mathrm{H} & -2.62326800 & 2.33665900 & -0.93122700 \\ \mathrm{H} & -2.56522100 & 2.02220900 & 0.78468400 \\ \mathrm{H} & -1.17220400 & 2.71191400 & 0.00533000 \\ \mathrm{H} & 2.24704300 & 3.55377100 & -2.07910200 \\ \mathrm{H} & 3.83370600 & 2.88867000 & -1.69117000 \\ \mathrm{H} & 2.85559300 & 2.15616500 & -2.97604800 \\ \mathrm{H} & 1.48852300 & 3.76056800 & 1.43692900 \\ \mathrm{H} & 1.05707300 & -2.42908100 & -2.40598000 \\ \mathrm{H} & -0.27985100 & -1.34776000 & -2.83992300 \\ \mathrm{H} & 1.38476000 & -0.77988900 & -2.89334900 \\ \mathrm{H} & 4.63513800 & -1.50338600 & 3.18563600 \\ \mathrm{H} & 3.66444800 & -0.03977400 & 3.45189900 \\ \mathrm{H} & 4.59384200 & -0.21596300 & 1.95864800\end{array}$

(2). Conformer2, Pop=48.11\% B3LYP/6-311++g(2d,p), E= -1497.71722039 au 62

$\begin{array}{lrrc}\text { C } & -3.72101700 & -0.12330800 & 2.02801400 \\ \mathrm{C} & -4.74787500 & 0.58630500 & 1.23752900 \\ \mathrm{O} & -4.59729800 & 0.57175500 & -0.11227800 \\ \mathrm{C} & -3.76611300 & -0.45110100 & -0.75656800 \\ \mathrm{C} & -2.36597700 & -0.58143800 & -0.03035300 \\ \mathrm{C} & -2.62162500 & -0.62556100 & 1.46113500 \\ \mathrm{C} & -1.72214200 & -1.92146700 & -0.47332000 \\ \mathrm{C} & -0.23598200 & -1.99031300 & -0.16136700 \\ \mathrm{C} & 0.54781100 & -0.90000200 & -0.91279700 \\ \mathrm{C} & -0.15947300 & 0.44703100 & -0.79208100 \\ \mathrm{C} & -1.41747900 & 0.61419700 & -0.32745400 \\ \mathrm{C} & 1.99357800 & -0.75742400 & -0.24128600 \\ \mathrm{C} & 2.81017800 & 0.39829300 & -0.84764000 \\ \mathrm{C} & 2.10612600 & 1.75864800 & -0.89456200 \\ \mathrm{C} & 0.64063900 & 1.59425800 & -1.37329500 \\ \mathrm{C} & 1.89295200 & -0.54860900 & 1.28061000 \\ \mathrm{C} & 2.70714100 & -1.63080900 & 1.97947300 \\ \mathrm{O} & 3.13337300 & -2.51699700 & 0.92839500 \\ \mathrm{C} & 2.76616900 & -2.09186200 & -0.31143000 \\ \mathrm{O} & -5.65010300 & 1.21780300 & 1.73282200\end{array}$




\begin{tabular}{|c|c|c|c|}
\hline $\mathrm{C}$ & -4.58674500 & -1.75341000 & -0.70806200 \\
\hline $\mathrm{C}$ & -3.66035700 & 0.01433300 & -2.20813600 \\
\hline $\mathrm{O}$ & 3.04441200 & -2.73540900 & -1.28223300 \\
\hline $\mathrm{O}$ & 1.28610100 & 0.30822700 & 1.86854000 \\
\hline $\mathrm{C}$ & 4.03979000 & 0.20736400 & -1.32980100 \\
\hline $\mathrm{C}$ & -1.96111000 & 2.00124300 & -0.03648400 \\
\hline $\mathrm{C}$ & 2.12530300 & 2.52279800 & 0.44462700 \\
\hline $\mathrm{C}$ & 2.80332100 & 2.74313600 & -1.87071500 \\
\hline $\mathrm{O}$ & 1.28277900 & 3.30689100 & 0.79814500 \\
\hline $\mathrm{O}$ & 3.27523300 & 2.33756300 & 1.13466800 \\
\hline $\mathrm{C}$ & 0.69838600 & -1.28733500 & -2.40671900 \\
\hline $\mathrm{C}$ & 3.89890700 & -1.09187900 & 2.75898300 \\
\hline $\mathrm{H}$ & -3.88653400 & -0.14136300 & 3.09901600 \\
\hline $\mathrm{H}$ & -1.84570700 & -1.06068500 & 2.08548200 \\
\hline $\mathrm{H}$ & -1.86954200 & -2.06328400 & -1.54623500 \\
\hline $\mathrm{H}$ & -2.23519500 & -2.75287900 & 0.01573300 \\
\hline $\mathrm{H}$ & -0.10045200 & -1.88202400 & 0.91942300 \\
\hline $\mathrm{H}$ & 0.15118100 & -2.97918800 & -0.42562500 \\
\hline $\mathrm{H}$ & 0.13611500 & 2.54612100 & -1.24044500 \\
\hline $\mathrm{H}$ & 0.68692000 & 1.43279100 & -2.45707000 \\
\hline $\mathrm{H}$ & 2.04523400 & -2.20101300 & 2.63925400 \\
\hline $\mathrm{H}$ & -4.17014700 & -2.52347700 & -1.35839200 \\
\hline $\mathrm{H}$ & -5.59671300 & -1.53202900 & -1.05795700 \\
\hline $\mathrm{H}$ & -4.66260900 & -2.16067600 & 0.30120200 \\
\hline $\mathrm{H}$ & -4.66258200 & 0.10400700 & -2.63154400 \\
\hline $\mathrm{H}$ & -3.16998200 & 0.98158600 & -2.29370700 \\
\hline $\mathrm{H}$ & -3.10381500 & -0.70575800 & -2.81102100 \\
\hline $\mathrm{H}$ & 4.61664600 & 1.01855000 & -1.75395600 \\
\hline $\mathrm{H}$ & 4.52000400 & -0.76239400 & -1.34075300 \\
\hline $\mathrm{H}$ & -2.57722500 & 2.40791400 & -0.84350500 \\
\hline $\mathrm{H}$ & -2.59121400 & 1.99122700 & 0.85141900 \\
\hline $\mathrm{H}$ & -1.15971200 & 2.70618800 & 0.17250300 \\
\hline $\mathrm{H}$ & 2.21202900 & 3.65770600 & -1.94820800 \\
\hline $\mathrm{H}$ & 3.80373300 & 3.02108600 & -1.53602000 \\
\hline $\mathrm{H}$ & 2.88095600 & 2.29896700 & -2.86511200 \\
\hline $\mathrm{H}$ & 3.22793800 & 2.90325300 & 1.92157400 \\
\hline $\mathrm{H}$ & 1.03833600 & -2.31493100 & -2.52273400 \\
\hline $\mathrm{H}$ & -0.26082800 & -1.17574000 & -2.91656000 \\
\hline $\mathrm{H}$ & 1.42017600 & -0.65463000 & -2.92333700 \\
\hline $\mathrm{H}$ & 4.45738200 & -1.91733700 & 3.20404200 \\
\hline $\mathrm{H}$ & 3.54555400 & -0.43649700 & 3.55756000 \\
\hline $\mathrm{H}$ & 4.56764400 & -0.52267800 & 2.11071700 \\
\hline
\end{tabular}

(3). Conformer3, Pop $=0.93 \%$ B3LYP/6-311++g(2d,p), $E=-1497.71350016$ au 


\begin{tabular}{|c|c|c|c|}
\hline $\mathrm{C}$ & 3.88466400 & -0.14971500 & -1.91784100 \\
\hline $\mathrm{C}$ & 4.87169600 & -0.70120300 & -0.96987700 \\
\hline $\mathrm{O}$ & 4.54216600 & -0.64590800 & 0.34842200 \\
\hline $\mathrm{C}$ & 3.51971700 & 0.29537900 & 0.83348700 \\
\hline $\mathrm{C}$ & 2.22007600 & 0.13888900 & -0.06306200 \\
\hline $\mathrm{C}$ & 2.66899900 & 0.22466300 & -1.51440400 \\
\hline $\mathrm{C}$ & 1.59352000 & -1.28264000 & 0.06273700 \\
\hline $\mathrm{C}$ & 0.59584900 & -1.45511700 & 1.20646700 \\
\hline $\mathrm{C}$ & -0.50449600 & -0.37437700 & 1.29776000 \\
\hline $\mathrm{C}$ & -0.04300800 & 0.97076500 & 0.75337800 \\
\hline $\mathrm{C}$ & 1.15423100 & 1.22138300 & 0.18565900 \\
\hline $\mathrm{C}$ & -1.82191700 & -0.78392600 & 0.46650500 \\
\hline $\mathrm{C}$ & -2.90961400 & 0.30229700 & 0.56867400 \\
\hline $\mathrm{C}$ & -2.46852500 & 1.74235100 & 0.27051200 \\
\hline $\mathrm{C}$ & -1.08865700 & 2.05317500 & 0.91661800 \\
\hline $\mathrm{C}$ & -1.50838900 & -1.05153100 & -1.01142900 \\
\hline $\mathrm{C}$ & -1.84518100 & -2.50286100 & -1.33301500 \\
\hline $\mathrm{O}$ & -2.31260700 & -3.06410400 & -0.09319700 \\
\hline $\mathrm{C}$ & -2.33638100 & -2.16187900 & 0.92737100 \\
\hline $\mathrm{O}$ & 5.89462400 & -1.24577100 & -1.30801500 \\
\hline $\mathrm{C}$ & 3.33518000 & -0.11937800 & 2.29174900 \\
\hline $\mathrm{C}$ & 4.16334900 & 1.68880300 & 0.80717600 \\
\hline $\mathrm{O}$ & -2.71046000 & -2.48564300 & 2.01742100 \\
\hline $\mathrm{O}$ & -1.08154200 & -0.27355600 & -1.82611300 \\
\hline $\mathrm{C}$ & -4.16243000 & -0.00183800 & 0.91230900 \\
\hline $\mathrm{C}$ & 1.45620900 & 2.60945600 & -0.36729300 \\
\hline $\mathrm{C}$ & -2.41200800 & 2.06121800 & -1.23849300 \\
\hline $\mathrm{C}$ & -3.47826600 & 2.78700700 & 0.81716600 \\
\hline $\mathrm{O}$ & -1.65172200 & 2.84360200 & -1.75081900 \\
\hline $\mathrm{O}$ & -3.41741900 & 1.47869300 & -1.92936000 \\
\hline $\mathrm{C}$ & -0.88628200 & -0.24328700 & 2.79654700 \\
\hline $\mathrm{C}$ & -2.88769000 & -2.65767400 & -2.43114500 \\
\hline $\mathrm{H}$ & 4.18233600 & -0.14539000 & -2.95989800 \\
\hline $\mathrm{H}$ & 1.92242500 & 0.53606000 & -2.24024800 \\
\hline $\mathrm{H}$ & 1.10715600 & -1.50836300 & -0.88930200 \\
\hline $\mathrm{H}$ & 2.39565800 & -2.01820400 & 0.16012200 \\
\hline $\mathrm{H}$ & 0.14839600 & -2.45312000 & 1.14458300 \\
\hline $\mathrm{H}$ & 1.12668300 & -1.45429600 & 2.15671100 \\
\hline $\mathrm{H}$ & -0.74808800 & 3.00481500 & 0.52010500 \\
\hline $\mathrm{H}$ & -1.26795700 & 2.21583700 & 1.98429700 \\
\hline $\mathrm{H}$ & -0.92397600 & -3.02965100 & -1.60460800 \\
\hline $\mathrm{H}$ & 2.52269700 & 0.44067500 & 2.75832900 \\
\hline $\mathrm{H}$ & 4.25367300 & 0.08575300 & 2.84472800 \\
\hline
\end{tabular}




$\begin{array}{lrrr}\mathrm{H} & 3.13730700 & -1.18600700 & 2.38339900 \\ \mathrm{H} & 5.11912900 & 1.63191700 & 1.33154400 \\ \mathrm{H} & 4.36003600 & 2.04638300 & -0.20282500 \\ \mathrm{H} & 3.54120000 & 2.42286000 & 1.32021700 \\ \mathrm{H} & -4.93341600 & 0.75429000 & 0.97714800 \\ \mathrm{H} & -4.47411300 & -1.00804000 & 1.15936700 \\ \mathrm{H} & 1.53920500 & 3.36249700 & 0.42246400 \\ \mathrm{H} & 2.39136400 & 2.63180500 & -0.92241300 \\ \mathrm{H} & 0.66973100 & 2.93795100 & -1.04829400 \\ \mathrm{H} & -3.07459400 & 3.79172400 & 0.67513000 \\ \mathrm{H} & -4.44151300 & 2.74166400 & 0.30784700 \\ \mathrm{H} & -3.64255000 & 2.63091600 & 1.88517400 \\ \mathrm{H} & -3.34327400 & 1.78076800 & -2.84822000 \\ \mathrm{H} & -1.03476100 & -1.22371300 & 3.24646700 \\ \mathrm{H} & -0.07322800 & 0.25953300 & 3.32735800 \\ \mathrm{H} & -1.80230300 & 0.32300600 & 2.95983500 \\ \mathrm{H} & -3.10756000 & -3.71437400 & -2.59234100 \\ \mathrm{H} & -2.50389300 & -2.23279600 & -3.36064200 \\ \mathrm{H} & -3.81395800 & -2.14276200 & -2.16807300\end{array}$




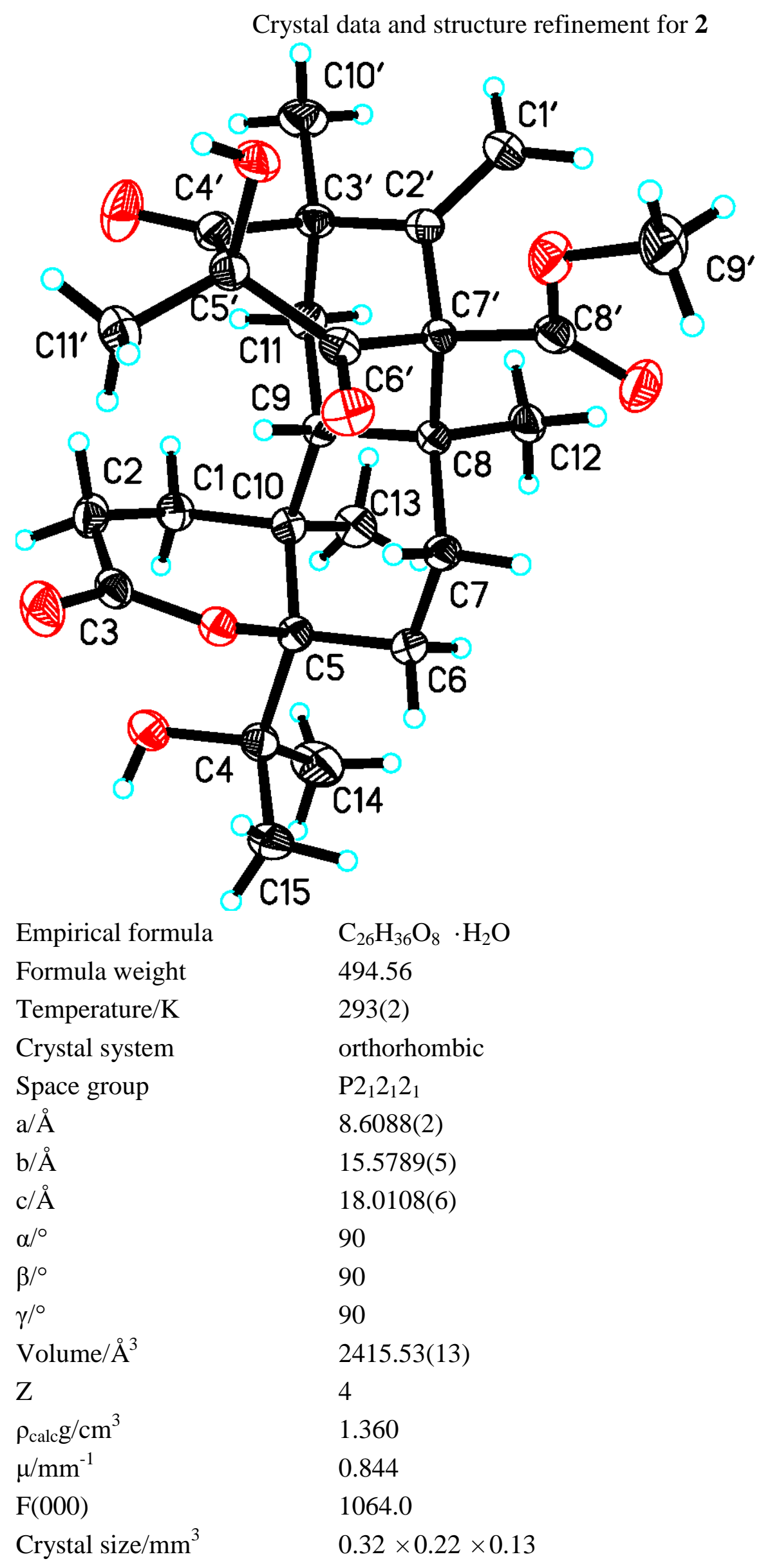


Radiation $\quad \mathrm{CuK \alpha}(\lambda=1.54184)$

$2 \Theta$ range for data collection $/{ }^{\circ} 7.502$ to 133.928

Index ranges $\quad-9 \leq \mathrm{h} \leq 10,-18 \leq \mathrm{k} \leq 18,-21 \leq 1 \leq 19$

Reflections collected $\quad 15377$

Independent reflections $\quad 4282\left[\mathrm{R}_{\text {int }}=0.0385, \mathrm{R}_{\text {sigma }}=0.0300\right]$

Data/restraints/parameters 4282/0/336

Goodness-of-fit on $\mathrm{F}^{2} \quad 1.064$

Final $\mathrm{R}$ indexes $[\mathrm{I}>=2 \sigma(\mathrm{I})] \quad \mathrm{R}_{1}=0.0403, \mathrm{wR}_{2}=0.0979$

Final $\mathrm{R}$ indexes [all data] $\quad \mathrm{R}_{1}=0.0476, \mathrm{wR}_{2}=0.1053$

Largest diff. peak/hole / e $\AA^{-3} 0.15 /-0.24$

Flack parameter $\quad 0.02(12)$

Hooft parameter $\quad 0.04(9)$ 


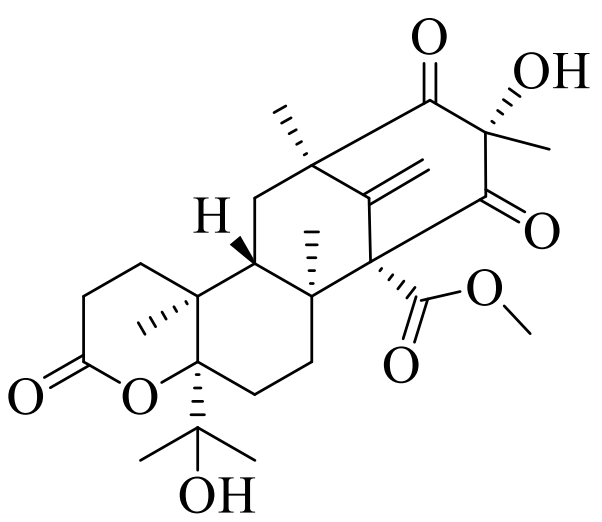

$2 a$

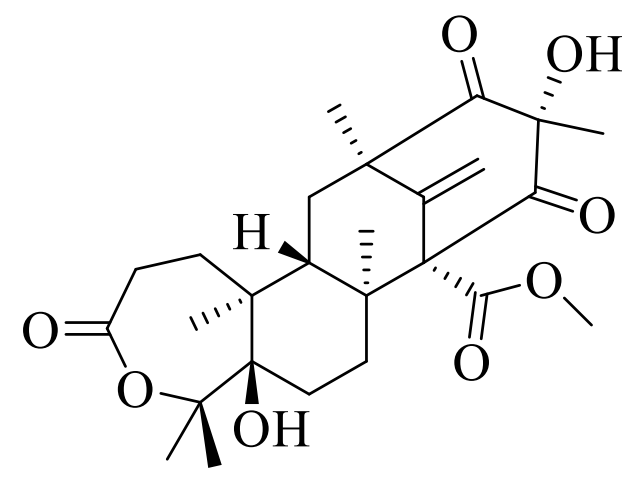

2b

Figure S25. Possible structures of $\mathbf{2}$ (2a and $\mathbf{2 b}$ ). 